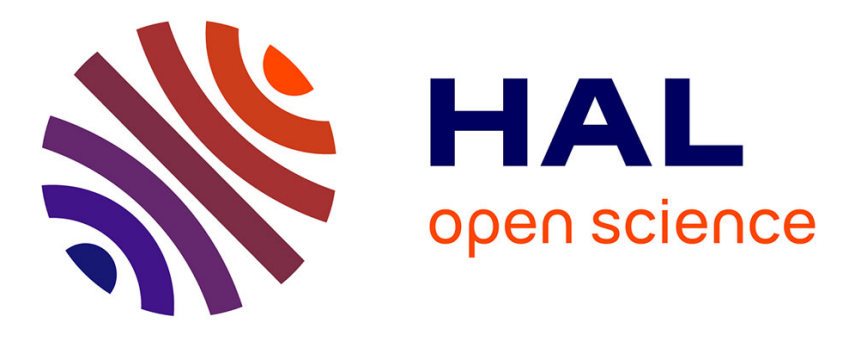

\title{
High Order X-FEM and Levelsets for Complex Microstructures: Uncoupling Geometry and Approximation
}

\author{
Grégory Legrain, Nicolas Chevaugeon, Kristell Dréau
}

\section{To cite this version:}

Grégory Legrain, Nicolas Chevaugeon, Kristell Dréau. High Order X-FEM and Levelsets for Complex Microstructures: Uncoupling Geometry and Approximation. Computer Methods in Applied Mechanics and Engineering, 2012, 241-244, pp.172-189. 10.1016/j.cma.2012.06.001 . hal-00703239

\section{HAL Id: hal-00703239 \\ https://hal.science/hal-00703239}

Submitted on 1 Jun 2012

HAL is a multi-disciplinary open access archive for the deposit and dissemination of scientific research documents, whether they are published or not. The documents may come from teaching and research institutions in France or abroad, or from public or private research centers.
L'archive ouverte pluridisciplinaire HAL, est destinée au dépôt et à la diffusion de documents scientifiques de niveau recherche, publiés ou non, émanant des établissements d'enseignement et de recherche français ou étrangers, des laboratoires publics ou privés.

\section{(ㄷ)(1) $\$$}

Distributed under a Creative Commons Attribution - NonCommerciall 4.0 International 


\section{GeM Institute}

GeM Institute UMR CNRS 6183

LUNAM Université

École Centrale de Nantes / Université de Nantes / CNRS,

1 Rue de la Noë, BP92101, 44321 Nantes, France.
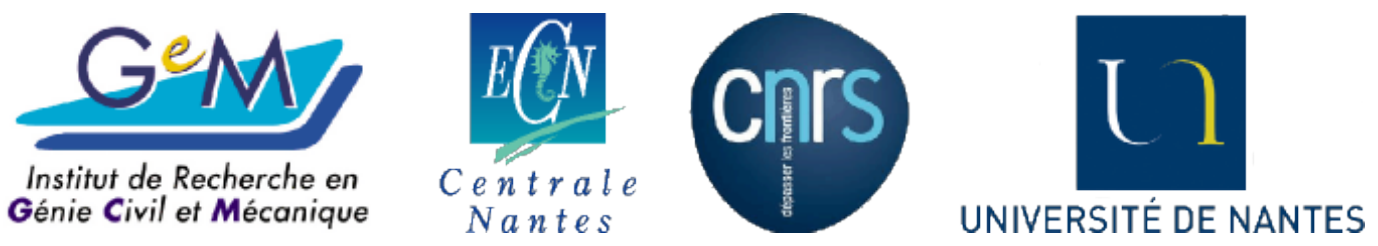

\section{High Order X-FEM and Levelsets for Complex Microstructures: Uncoupling Geometry and Approximation}

G. Legrain, N. Chevaugeon, K.Dréau

Preprint submitted to:

Computer Methods in Applied Mechanics and Engineering 


\title{
High Order X-FEM and Levelsets for Complex Microstructures: Uncoupling Geometry and Approximation
}

\author{
G. Legrain, N. Chevaugeon, K.Dréau
}

SUMMARY

\begin{abstract}
In this contribution, a strategy is proposed for uncoupling geometrical description and approximation with the X-FEM. The strategy is based on an uniform coarse mesh that defines a high order approximation of the mechanical fields and an adapted mesh that defines the geometrical features by means of levelsets. The connection between the geometry and the approximation is obtained by sharing the quadtree trees of the two meshes. Numerical examples involving level-set based parts, convergence studies, mechanical computations and numerical homogenization show good promise for this approach.
\end{abstract}

Preprint submitted to: Computer Methods in Applied Mechanics and Engineering KEY WORDS: Partition of Unity, X-FEM, High Order, Image-based Computations, Nitsche's Method

\section{Introduction}

Since the beginning of finite elements, numerous researches have been carried out in order to build meshes containing elements with geometrically correct shapes [1]. In the two dimensional setting, the construction of such meshes is well mastered for complex geometries for both triangular and quadrangular elements. However, generating meshes for complex structures and geometrical singularities is still awkward in 3D, especially for hexaedral meshes [2]. This is why new strategies have been proposed in order to alleviate these meshing issues. Approaches such as fictitious domains propose to approximate the mechanical fields on a grid in which the physical domain is embedded [3]. Finite Differences, Finite Elements or Finite Volumes can then be used for the approximation. Difficulties arise when imposing the boundary conditions (especially Dirichlet): Ad-hoc approaches such as Lagrange multipliers or penalty methods have to be considered [4]. Moreover the treatment of material interfaces need special formulations $[5,6]$ if one wants to keep optimal converge properties. Recently, Düster et al. $[7,8]$ have proposed a high order extension of the fictitious domain approach coined Finite Cell method. This approach allows to obtain accurate results on domains defined independently from the discretization. However, the case of material interfaces seems more difficult to take into account: Adapted approaches have to be considered in order to avoid pathological oscillations near interfaces, especially in large strain $[9,10]$. In addition interfaces are 
fuzzy, so that the geometrical description of the domain of interest is dependent on the resolution of the integration cells and in the number of integration points. Meshless methods are also an answer to the meshing issue, as the connectivity is obtained by means of domains of influence that are not mesh based (see [11] for a review on this topic). Moreover, the regularity of the shape functions is higher than low-order finite element ones. However, the computational cost of these approaches is still higher than finite elements, and the parameters involved in the formulation are not always easy to select a-priori. In the last ten years, partition of unity finite element methods have proved to be an effective answer for alleviating meshing constraints and improving the accuracy of the solution by means of the physical knowledge of its behaviour. The two main partition of unity finite elements methods are the Generalized Finite Element Method (GFEM) $[12,13]$ and the eXtended Finite Element Method (X-FEM) [14]. Both methods use the partition of unity to enrich the underlying finite element approximation with adapted enrichment functions. While the geometry is defined explicitly in the GFEM, it is defined implicitly with the X-FEM by means of Level Sets [15]. Both approaches were applied in a wide variety of applications from fracture mechanics $[16,17,18]$ to the treatment of physical interfaces $[19,20,21,22]$. The reader can refer to two recent reviews on this topic $[23,24]$. One objective within these approaches is to represent the geometry independently of the approximation. Attempts have already been made in order to try to dissociate these quantities. Earlier works conducted by Strouboulis et al. [12] considered the material interfaces explicitly which enabled to define their degree of smoothness independently of the density of the mesh used for the approximation. A similar approach was also considered by Peirera et al. [18] in the context of 3D fracture mechanics where the authors proposed strategies for the generation of the sub-elements that are needed for the integration of the weak form. In the context of the Finite Cell Method, Düster et al. $[7,8]$ proposed an integration of the weak form on a set of cubic sub-cells that approximates the geometrical interfaces as "voxelized" surfaces (with a selective use of the integration points). The idea of defining the approximation on a coarse mesh with high order hierarchical polynomials seems to be promising, provided that the interfaces are correctly represented. Here, the objective is to use a high order approximation and a $C^{0}$ representation of the interface as in $[12,18]$, but with an implicit representation of the interfaces which leads to extra difficulties. In addition, focus is put on the optimal convergence of the approach. This aspect has already been studied in the context of high order X-FEM by Dréau et al. [25] and Cheng at al. [26]. Earlier attempts were proposed in the context of fracture mechanics by Prabel et al. [27] where the levelset was defined on an auxiliary mesh, in order to improve the efficiency of the levelset propagation process. This approach was also considered by Rannou et al [28] to define the levelset of a crack surface by means of micro CT. However, the levelset is still projected on the computational mesh which can lead to severe geometrical loss if this mesh is too coarse. Two different path were followed in [25] and [26]. In [26], the authors considered a high order interpolation for the levelsets and a high-order mapping for the integration elements. However, the extension of the approach to 3D with complex interfaces seems tricky, especially if the order of the levelset is increased. On the contrary, in was chosen in [25] to keep a low-order interpolation for the levelset, but on an extremely fine mesh 
which makes the approach easy to extend in 3D. The objective of this contribution is to take advantage of this approach, not only for the improved convergence properties, but also to be able to solve mechanical problems with complex geometries (such as those that can be encountered in image-based analysis). Because of this application field, a different implementation is proposed: it is based on the use of two meshes (one for the geometry and one for the approximation). The method relies on a quadtree database that is used to generate both geometrical and approximation meshes, but also for the necessary communications between them. Stress is also put on the optimal convergence of the approach for material interfaces, which was not obtained in [25]. This is why a Heaviside enrichment is considered for material interfaces, together with the use of Nitsche's method [29, 30, 31, 32] to cancel the displacement jump. This contribution is organized as follows: In a first part, the X-FEM is recalled. Then, an approach for separating geometrical representation and field approximation is presented. The use of the Nitsche approach is then presented for the treatment of material interfaces. Finally, the strategy is validated with some numerical examples consisting of convergence studies, high order micro-mechanical computations defined on image acquisition and mechanical parts.

\section{The eXtended Finite Element Method and LevelSets}

\subsection{The X-FEM}

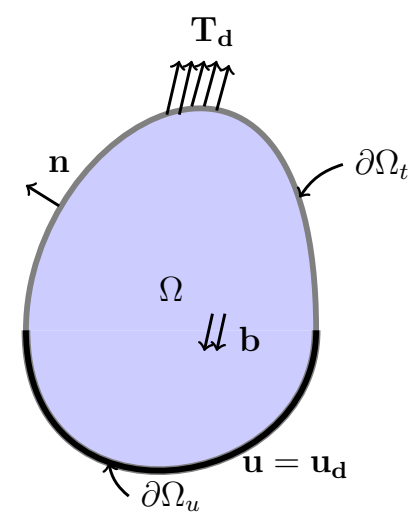

Figure 1: Problem of interest.

Consider the static response of a two dimensional elastic body which occupies a bounded domain $\Omega$ with a sufficiently smooth boundary $\partial \Omega$ split into two disjoint parts: $\partial \Omega_{u}$ where displacements are prescribed (Dirichlet boundary conditions) and $\partial \Omega_{t}$ where tractions are prescribed (Neumann boundary conditions) (see Figure 1). The body is 
initially in an undeformed and stress-free configuration. The governing equations are:

$$
\begin{cases}\operatorname{div} \underline{\underline{\sigma}}+\mathbf{b}=\mathbf{0} & \text { in } \Omega \\ \underline{\underline{\varepsilon}}=\frac{\overline{1}}{2}\left(\underline{\left.\underline{\nabla} \mathbf{u}+\underline{\underline{\nabla}} \mathbf{u}^{T}\right)}\right. & \text { in } \Omega \\ \underline{\underline{\underline{\sigma}}} \cdot \mathbf{n}=\mathbf{T}_{\mathbf{d}} & \text { on } \partial \Omega_{t} \\ \mathbf{u}(\mathbf{x})=\mathbf{u}_{\mathbf{d}} & \text { on } \partial \Omega_{u} \\ \underline{\underline{\sigma}}=\mathcal{C}: \underline{\underline{\varepsilon}} & \text { in } \Omega\end{cases}
$$

where $\underline{\sigma}$ is the Cauchy stress tensor, $\mathbf{b}$ is the body force, $\mathbf{u}_{\mathbf{d}}$ is the prescribed displacement field, $\overline{\overline{\mathbf{T}}}_{\mathbf{d}}$ are the prescribed tractions, $\mathbf{n}$ is the outward unit normal to the boundary $\partial \Omega, \underline{\underline{\varepsilon}}$ is the linearized strain tensor and $\mathcal{C}$ is the fourth-order elasticity tensor. For a linear isotropic elastic material, the constitutive equation only depends on two scalar parameters $\lambda$ and $\mu$ (the Lamé coefficients) and can be written as:

$$
\underline{\underline{\sigma}}=\lambda \operatorname{Tr}(\underline{\underline{\varepsilon}})(\mathbf{u}) \underline{\underline{I}}+2 \mu \underline{\underline{\varepsilon}}(\mathbf{u})
$$

The above strong form of the governing equation (1) can be expressed into the following weak form:

$$
\int_{\Omega} \mathbf{b} \cdot \mathbf{v} d V+\int_{\partial \Omega_{t}} \mathbf{T}_{d} \cdot \mathbf{v} d A=\int_{\Omega} \underline{\underline{\varepsilon}}(\mathbf{v}): \underline{\underline{\sigma}}(\mathbf{u}) d V \quad \forall \mathbf{v} \in H^{1}(\Omega) \mid \mathbf{v}=\mathbf{0} \text { on } \partial \Omega_{u}
$$

where the linear (resp. bilinear) form in the left hand side (resp. right hand side) of this expression will be denoted as $l(\mathbf{v})$ (resp. $a(\mathbf{u}, \mathbf{v})$ ). The weak formulation (3) is solved numerically by considering the approximation of both displacement and test fields. Within classical finite elements, the approximation of the vector field $\mathbf{u}(\mathbf{x})$ on an element $\Omega_{e}$ is:

$$
\left.\mathbf{u}(\mathbf{x})\right|_{\Omega_{e}}=\sum_{\alpha=1}^{n} u^{\alpha} \mathbf{N}^{\alpha}(\mathbf{x})
$$

where $n$ is the number of coefficients describing the approximation over the element, $u^{\alpha}$ is the $\alpha^{\text {th }}$ nodal value of this approximation and $\mathbf{N}^{\alpha}$ is the classical vectorial shape function associated with degree of freedom $u^{\alpha}$. Within the partition of unity [33], the approximation can be enriched as:

$$
\left.\mathbf{u}(\mathbf{x})\right|_{\Omega_{e}}=\sum_{\alpha=1}^{n} \mathbf{N}^{\alpha}\left(u^{\alpha}+\sum_{\beta=1}^{n_{e}} a_{\beta}^{\alpha} \varphi_{\beta}(\mathbf{x})\right)
$$

where $n_{e}$ is the number of enrichment modes, $a_{\beta}^{\alpha}$ is the additional degree of freedom (dof in the following) associated to dof $\alpha$ and $\varphi_{\beta}$ stands for the $\beta^{\text {th }}$ scalar enrichment function. The number and the expression of the enrichment functions vary with the problem to model. In the case of holes and material inclusions, they allow the use of a non-conforming mesh: a regular mesh is constructed, and the presence of internal boundaries such as inclusions is handled by means of the enrichment functions. In addition, only a few degrees of freedom are added by the enrichment as this enrichment is local: in the case of material interfaces, only the nodes whose support is cut by the interface are enriched [19]. Finally, it is to note that the modified Gauss quadrature scheme described in [14] is used to integrate the weak form, as the gradient of the enriched part of the approximation is discontinuous. 


\subsection{Coupling X-FEM with levelsets}

High-order levelsets Since the work of Stolarska et al. [34], the X-FEM has been coupled with the levelset approach [15] in order to track the position of the interfaces on the mesh. The levelset values are interpolated on the finite element mesh, and the location of the interface is obtained by looking for the iso-zero of the levelset. Initially, linear interpolation of the levelset was considered until the work of Budyn et al. [35] where a quadratic interpolation of the levelset was used. Later works conducted by Legay et al. [36], Sukumar et al. [37] and Cheng and Fries [26] recently used a high order interpolation of the levelset. In [26], the authors focused on the search for optimal convergence rates for both strong and weak discontinuities. Special high-order sub-elements were introduced in order to integrate the weak form for a high order interpolation of the levelset (see figure 2), and the corrected X-FEM was considered to recover close-to-optimal convergence rates. This approach is appealing and leads to a very good accuracy. However, it seems difficult to generalize to complex levelset shapes, especially in 3D. For example, a third order approximation of the level-set can define non-convex iso-zero lines (in 2D) that could lead to difficulties in the definition of the sub-elements. In addition, this approach requires the knowledge of the correct location of the nodes of the sub-element on the iso-zero. Finding the location of these points can be difficult for high order level sets: an efficient scheme was proposed in [38] for this purpose.

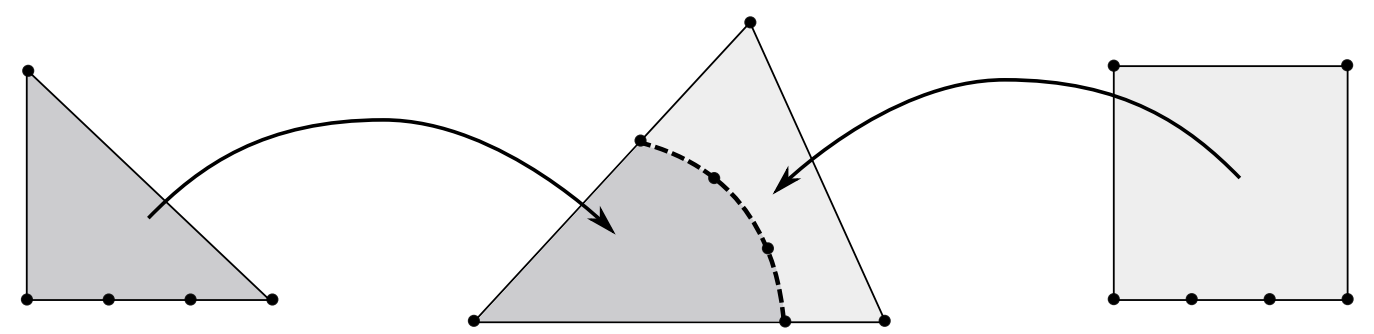

Figure 2: Improvement of the geometrical accuracy: Use of high order levelsets and special subelements.

Sub-Grid levelsets Another alternative possibility was proposed in [25]: Rather than interpolating the levelset using high order shape functions, the authors proposed to keep it linear (which enables to use efficient strategies for locating the interface), but on a highly refined sub-mesh around the interface (see figure 3) where the smaller elements near the interface represent the integration cells on which the levelset is interpolated (sub-grid levelset). This strategy is close to the one proposed by Parvizian et al. [7] in the context of the finite cell method. Thanks to this approach, arbitrary fine piecewise interfaces can be described on a coarse approximation mesh. However, this initial contribution was restricted to analytical levelsets only. The price to be paid for this improvement of the geometrical accuracy lies in the integration of the weak form that need numerous sub-elements, and in the use of an additional parameter which is the size 
of the sub-elements. It was seen that this approach leads to optimal convergence in the case of free surfaces, provided that the integration cells were small enough. However, the case of material interfaces was still problematic. Indeed, the ridge function [20] cannot be used in this case. Recall the expression of this enrichment function:

$$
R(\mathbf{x})=\sum_{i}\left|\phi^{i}\right| N^{i}(\mathbf{x})-\left|\sum_{i} \phi^{i} N^{i}(\mathbf{x})\right|
$$

in this expression, $\phi^{i}$ stands for the value of the levelset at node $i$, and $N^{i}(\mathbf{x})$ stands for the shape function associated to node $i$. If both shape functions and levelset values are associated to the approximation mesh, then an inconsistency occurs between the geometry of the interface and the levelset function (the former depends on the coarse representation of the interface whereas the later depends on the fine one) see figure 4.a. If shape functions and levelset values are associated to the sub-grid levelset, then the inconsistency disappears (see figure 4.b). However in this case the enrichment function has a discontinuous slope not only across the interface, but also inside the mesh (lines $A B$ and $C D$ in figure 4.b). In [25], a methodology was proposed for the construction of ridge functions such as the one presented in figure 4.c. However, this definition leads to non-optimal convergence rates.
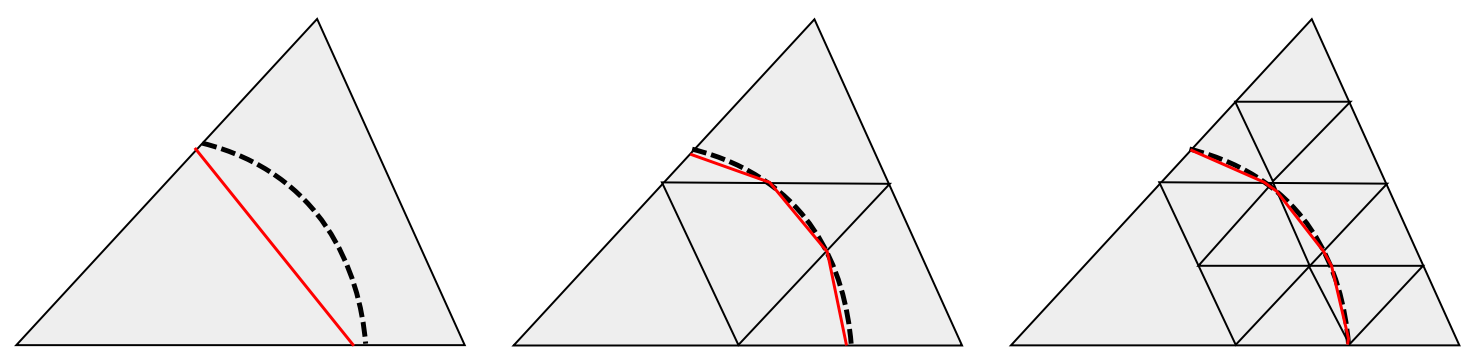

Figure 3: Improvement of the geometrical accuracy: The integration mesh is recursively refined around the interface.

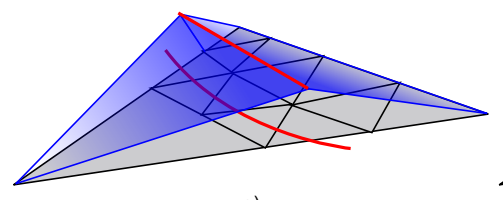

a)

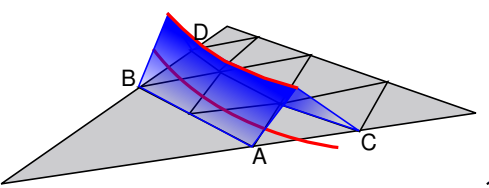

b)

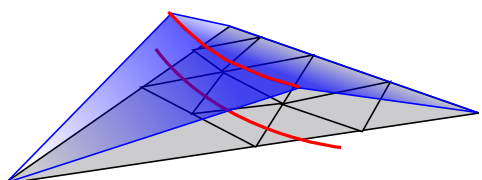

c)

Figure 4: Definition of the ridge enrichment function in the case of a sub-grid levelset. a) Ridge defined on the coarse mesh; b) Ridge defined on the sub-grid; b) Correct ridge definition. 


\section{Separating Geometry and Approximation}

Beside recovering optimal convergence rate, the objective of this contribution is to uncouple as much as possible the supports of geometry and approximation for application in image-based analysis. This allows to consider the full geometrical informations of the image and select the way the unknown field is interpolated (mesh, order) according to the available computational resources. This is the key difference with [39] and [20] where geometry and approximation are tied together. The geometrical input can be either an analytical levelset or the levelset segmentation of an image. In the latter case, the smallest size for elements of the geometrical mesh is given by the image (it is the size of a pixel). Thus, the additional size parameter introduced in [25] is known a priori. Following the strategy proposed in [39, 40,41,42,43], the geometrical informations segmented from the image are embedded into an octree mesh. Note that octree databases were already used for the GFEM [12], where the so-called pixelized integration was proposed. The key difference with the current approach lies in the fact that the accuracy of the integration is better: in each cell cut by the interface the integration rule is adapted, based on the levelset subelements. On the contrary, pixelized integration consider a cell as either inside or outside of the domain.

\subsection{Quadtree / Octree partition of space}

Quadtree (resp. Octree) is a spatial decomposition method that subdivides recursively the 2D (resp. 3D) bounding-box of the domain of interest $(\Omega)$ [1]. This box is first subdivided into four (resp. eight) equally-sized cells, each of which may be recursively subdivided several times (see figure.5(a)). The level of an element corresponds to the number of subdivisions required to obtain this cell from the bounding box. Note that the level of the root cell is zero (no subdivision was needed to obtain it). In order to manage mesh gradation, the level difference between adjacent cells may not exceed one. This is also called the 2:1 rule (any element have at most two neighbours along its edges (resp. four along its faces)). One key aspect of quadtree/octree structures is that all the topological informations can be stored as a tree, so that levels and neighbouring informations can be easily obtained (see figure.5(b)). Finally, note that the implementation used in this paper is based on a linear encoding of the tree [1].

\subsection{Construction of geometrical and approximation meshes}

The strategy for constructing both geometrical and approximation mesh is presented in figure 6: Two instances of the same quadtree database are considered: one defining the geometry and one defining the approximation. As we are only interested in the representation of the iso-zero of the levelset, only the cells of the quadtree that are crossed by the iso-zero need to be kept for the geometrical representation, and the other elements can be derefined (active geometrical cells are represented with lower script $\star$ in figure 6). This first instance of the quadtree is transformed into a so called geometrical finite element mesh by converting each quadtree cell into two triangular finite elements. 


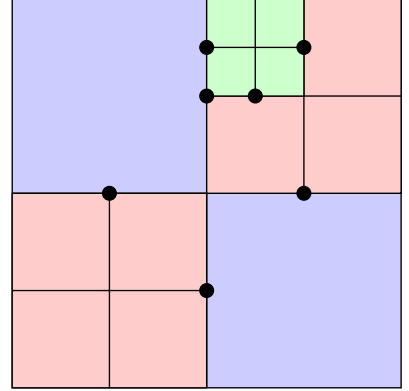

(a)

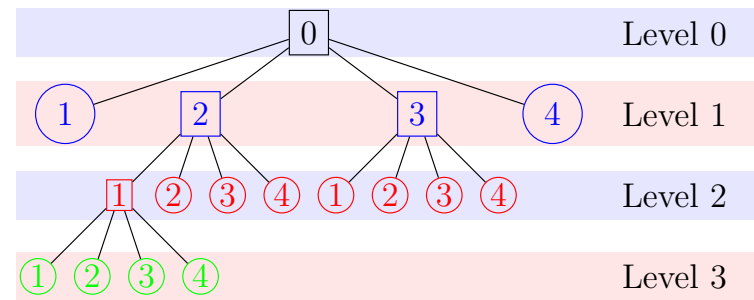

(b)

Figure 5: A quadtree mesh (a) and its representative tree (b). Hanging nodes are highlighted by $\bullet$. Note that in this case, the $2: 1$ rule is not verified.

Note that the 2:1 rule is not mandatory in this case, as no mechanical field is attached to the elements. On the other hand, a second instance of the same quadtree database is used to construct an approximation mesh (active approximation cells are represented with upper script $\bullet$ in figure 6 ). The information transfer between the geometry and the approximation is handled by the quadtree structure: an element in the geometrical mesh knows directly who is his father in the approximation mesh. The remaining of the strategy is similar to [25], except that the sub-mesh for integrating the weak form is directly the geometrical one when the element is crossed by an interface (otherwise its classical gauss points are used). Practically, no element is reconstructed in the sub-mesh of the approximation mesh: The pointers of the geometrical elements are simply copied in the approximation sub-mesh. Thanks to this approach a clear distinction is made between geometry and approximation which gives a wide flexibility to the user that can manage its geometrical model independently of the approximation. In this contribution, the use of high order hierarchical shape functions is considered for the approximation, which enables a high accuracy even with coarse approximation meshes. This strategy shares some similarities with the finite cell approach $[7,8]$, but with a more accurate integration process (no voxelization of the boundary as in [8]), and more flexibility thanks to the X-FEM (possibility to treat material interfaces, fracture mechanics problems).

\subsection{Quadtree partition of an implicit domain}

The construction of the geometrical mesh based on the levelset is now discussed. As noted in section 3.1 a linear encoding octree is implemented here, which means that the deepest level of the octree is known in advance. This implementation enables the use of a bottom-up strategy. Once the maximum level is known, the levelset can be evaluated at each corner of the higher level cells. From this initial evaluation, it is possible to test whether a cell at any level is cut or not by the interface (checking the minimum and maximum values of the levelset at the corners of the cell). Cut cells from the target level can thus be activated in the database. In a second step, the octree is coarsened from each inactive cell of the target level in order to construct a refined mesh near the interface. 


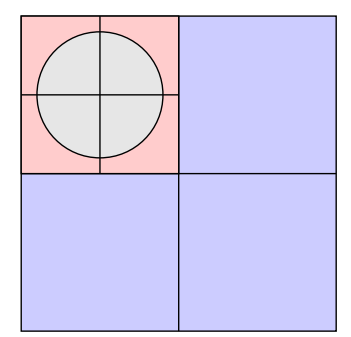

Geometrical cells

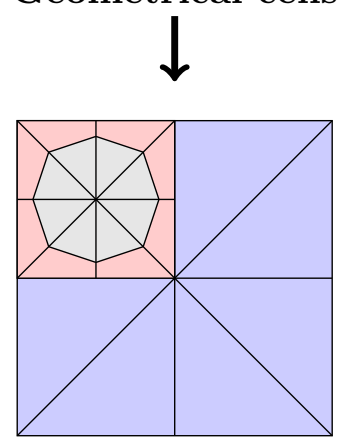

Geometrical mesh
Level

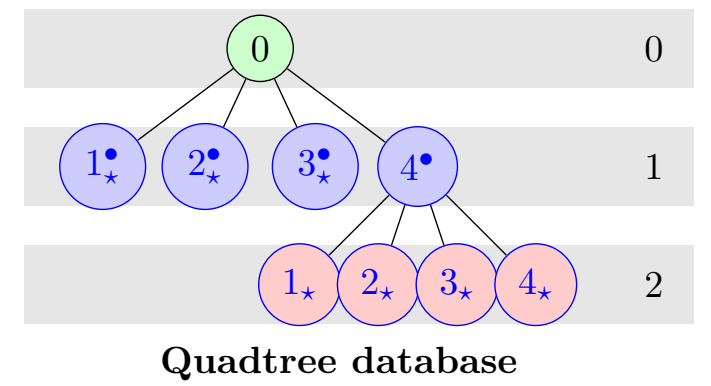

Quadtree database

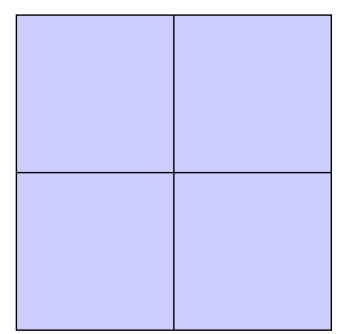

Approximation cells
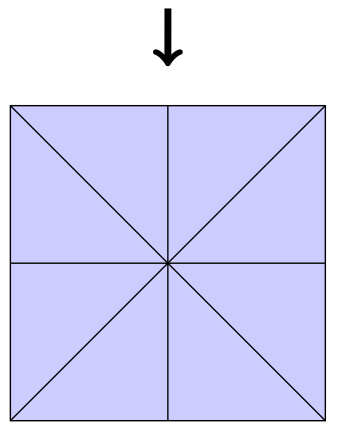

Approximation mesh

Figure 6: Two instances of the octree database for a square with a circular interface. Left: geometry, right: approximation, center octree database. Geometrical active cells are represented with lower script $\star$, approximation active cells with upper script • . In a last step, the cells are transformed into two triangular elements.

Finally, the 2:1 rule is prescribed by refining cells that violate this rule. The use of a top-down approach is also possible but more complicated. In this case, procedures adapted from the one presented in [44] can be considered to build the geometrical mesh.

\subsection{Recovering optimal convergence rate for material interfaces}

Enrichment As stated in section 2.2, the modification of the ridge function proposed in [25] could not allow optimal convergence rates. Here, an alternative approach is considered by means of the Nitsche approach $[29,30]$. This approach was extensively used in the context of both Meshless [45, 46] and X-FEM [30, 31, 32, 47] recently. In this case a Heaviside enrichment is used across the material interfaces, and the displacement jump is weakly canceled. It was proved in [48] that in the case of a stationary heat equation problem, optimal convergence could be obtained using this approach for any approximation order (and exact geometry). In the case of material interfaces, the weak form presented in (3) is modified as follows:

$$
a(\mathbf{u}, \mathbf{v})-\int_{\Gamma}\{\underline{\underline{\sigma}} \cdot \mathbf{n}\} \llbracket \mathbf{v} \rrbracket d \Gamma-\int_{\Gamma}\{\delta \underline{\underline{\sigma}} \cdot \mathbf{n}\} \llbracket \mathbf{u} \rrbracket d \Gamma+\beta \int_{\Gamma} \llbracket \mathbf{u} \rrbracket \llbracket \mathbf{v} \rrbracket d \Gamma=l(\mathbf{v})
$$


where $a(\mathbf{u}, \mathbf{v})$ and $l(\mathbf{v})$ are respectively the linear and bilinear forms introduced earlier. $\{\bullet\}$ is the average operator and $\llbracket \bullet \rrbracket$ is the jump operator. In this paper, $\{\bullet\}$ is defined as $1 / 2\left[\bullet\left(\mathbf{x}^{+}\right)+\bullet\left(\mathbf{x}^{-}\right)\right]$, and $\llbracket \bullet \rrbracket$ as $1 / 2\left[\bullet\left(\mathbf{x}^{+}\right)-\bullet\left(\mathbf{x}^{-}\right)\right]$where $\bullet\left(\mathbf{x}^{+}\right)$stands for the evaluation of $\bullet$ on the positive side of the interface (pointed by the normal vector) and $\bullet \cdot\left(\mathbf{x}^{-}\right)$for the evaluation of $\bullet$ on the negative side. As stated in $[30,49,32]$, the Nitsche approach can be viewed as a stabilized Lagrange multiplier approach (with condensed multiplier), and parameter $\beta$ plays role as stabilization parameter: Stability is ensured if the coercivity of the bilinear form is verified:

$$
\exists C_{I}>0 \text { such that }\|\{\underline{\underline{\sigma}}(\mathbf{v}) \cdot \mathbf{n}\}\|_{\Gamma} \leq C_{I}\|\mathbf{v}\|
$$

where $\|\bullet\|$ stands for the energetic norm defined by means of $a(\mathbf{u}, \mathbf{v})$, and $\|\bullet\|_{\Gamma}$ is defined as:

$$
\|\bullet\|=\int_{\Gamma} \bullet \bullet \cdot d \Gamma
$$

Nitsche's parameter $\beta$ can be approximated by solving a global eigenvalue problem, as proposed by Griebel [46]:

$$
[A]\{x\}=\Lambda[B]\{x\}
$$

where $\Lambda$ is the generalized eigenvalue associated to (10) with the following definitions:

$$
\begin{aligned}
& {[A]_{i j}=\int_{\Gamma}\left\{\underline{\underline{\sigma}}\left(\mathbf{N}^{i}\right) \cdot \mathbf{n}\right\} \cdot\left\{\underline{\underline{\sigma}}\left(\mathbf{N}^{j}\right) \cdot \mathbf{n}\right\} d \Gamma} \\
& {[B]_{i j}=\int_{\Omega} \underline{\underline{\varepsilon}}\left(\mathbf{N}^{i}\right): \mathcal{C}: \underline{\underline{\varepsilon}}\left(\mathbf{N}^{j}\right) d V}
\end{aligned}
$$

where $i, j$ represents the set of the shape functions whose support is cut by the interface. Note that $[B]$ can be extracted from the stiffness matrix of the system, and that $[A]$ only involves integrations along lines (in 2D) or surfaces (in $3 \mathrm{D}$ ). To ensure stability $\beta$ is taken as $2 \max (\Lambda)[46,31]$. Alternatively, Dolbow et al. proposed to define this parameter as a local quantity that evolves along the boundary [50,31]. Recently, a local eigenvalue approach was proposed in order to alleviate the computational cost introduced by the eigenvalue problem [32]. In following the numerical examples, $\beta$ will be computed by means of the global eigenvalue problem proposed by Griebel et al. otherwise stated. Moreover, the Heaviside function considered in the following corresponds to the sign of the levelset $( \pm 1)$.

Enrichment strategy When multiple interfaces cross the support of an entity (see for example figure 7), multiple enrichment functions have to be considered in order to preserve the convergence of the approximation. Indeed, if only one enrichment is considered for node $I$, the approximation is not rich enough to make displacement jump vanish along both interfaces. It was shown in Tran et al. [51] that an entity should be enriched with respect to all the interfaces crossing its support. In this case, the authors proposed to define separate enrichment functions for each of the interfaces. An improved 
approach was used here in order to decrease the number of enrichment functions when hundred of inclusions are considered: The set of inclusions is first "colored", so that they are classified in a small number of sets. Within each of these sets, the inclusions do not interact together (i.e. none of them cross the support of a common entity), so that they are independent in term of the enrichment process, and can share a common enrichment function (see figure 8). Thus, only inclusions belonging to two different sets influence common entities. Thanks to this approach, a maximum of 8 enrichment functions had to be considered in the numerical examples presented hereunder.

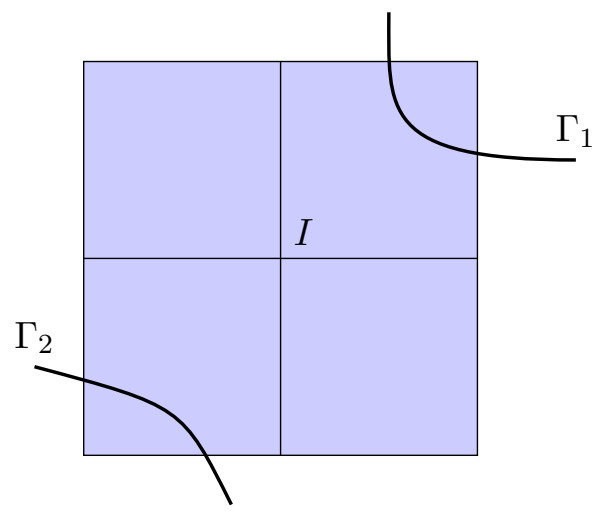

Figure 7: Support of a node $I$ crossed by two interfaces $\Gamma_{1}$ and $\Gamma_{2}$.

\subsection{Numerical cost of the approach}

As presented in section 2.2, the proposed approach introduces numerous integration cells in order to integrate the weak form on the fine geometry. This is why the additional cost of the approach lies in the integration procedure, especially for high-order polynomial approximations where more integration points are needed on each subelement. On the contrary, computational time is saved thanks to the small size of the stiffness matrix which lowers the time needed to solve the linear system. Moreover in the case of transient equations (such as linear heat equation), integration is done only once whereas the resolution of the linear system is done at each timestep. Nevertheless, the integration process need to be optimized in order to lower the impact of these additional costs. In this contribution, the assembly process was parallelized using multi-threading directives. Parallelizing the assembly is easy as no communications are involved during the process, leading to nice speedups. The other possibility would consist in vectorizing the assembly process, as proposed in [52]. We are currently improving the numerical efficiency of the proposed approach by replacing on the fly the integration cells by means of a local Delaunay tetrahedralization, following [18].

Concerning non-linear material behaviour, Nitsche's stabilization parameter should be recomputed at each iteration, which could be cumbersome. In this case, one could compute it using the local eigenvalue problem and keep the same value for $\beta$ during a sequence of iterations. Otherwise, Nitsche's method could be used as a penalty method 


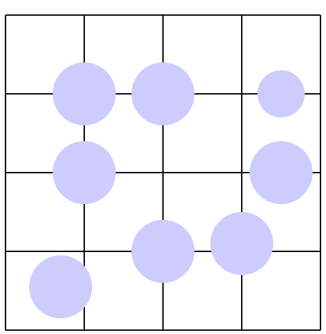

Mesh + inclusions

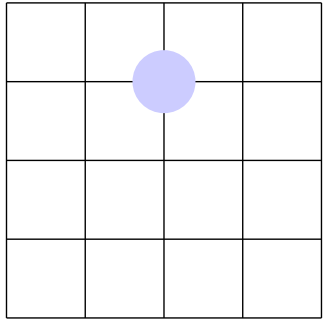

Inclusions set 3

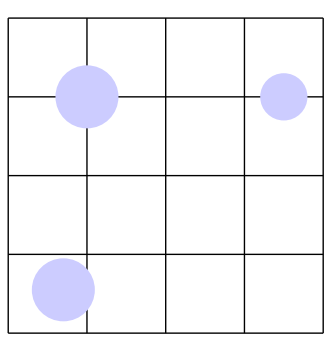

Inclusions set 1

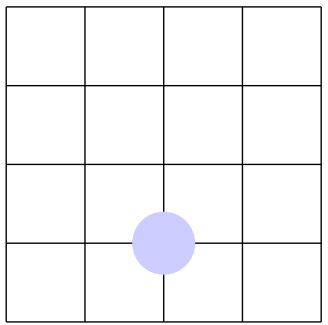

Inclusions set 4

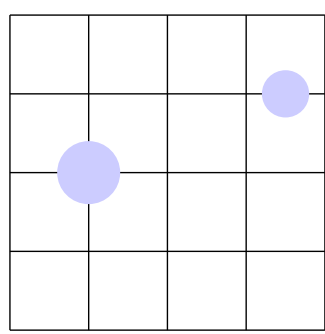

Inclusions set 2

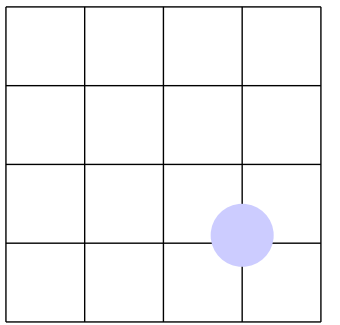

Inclusions set 5

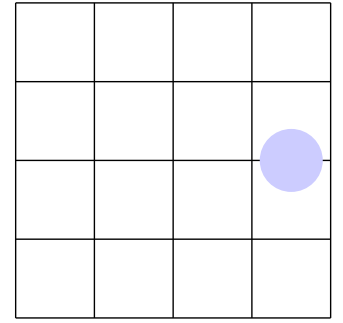

Inclusions set 6

Figure 8: Separation of the inclusions into independent sets. In this problem, 6 enrichments will be used, rather than 8 .

by fixing a-priori the value of $\beta$ : In this case, one gets a lower conditioning number as the method requires low stabilization values with respect to classical penalty (where the penalty parameter has to scale in $O\left(h^{-\frac{2 p+1}{3}}\right)$ for order $p$ elements ${ }^{1}$ ). Moreover, Nitsche's formulation is consistent which is not the case for the penalty approach.

\section{Numerical Examples}

The proposed approach is now validated on various numerical examples. First, the strategy is applied in the context of the simulation of levelset based parts. Second, the influence of the accuracy of the description of the interface (level of the octree near the material interface) is studied. Finally, the approach is applied to material homogenization, and the results are compared to classical X-FEM solutions. All these validations are based on the convergence of the global energy norm of the error in the domain. When an analytical solution is available, both local and global errors can be computed. Otherwise, following [53] the error in the strain energy is considered. In this case, no information can be obtained concerning the local errors unless a local error estimator is used.

\footnotetext{
${ }^{1}$ Note that for $p \geq 2$, optimal rates of convergence cannot be obtained because of the lack of consistency of the penalty method. In this case, the best convergence rate that could be obtained in the energy norm is only $O\left(h^{-\frac{2 p+1}{3}}\right)$, see [45]
} 


\subsection{Level Set parts}

The proposed approach is first illustrated in the case of the static behaviour of a mechanical part. The objective is to show how it can be used in order to totally free the approximation mesh from the geometry, as in the case of fictitious domain approaches. In this case, the approach is more related to the Finite Cell approach [7], but with a better representation of the interface. Consider the example depicted in figure 9 (a) inspired from the one proposed in [54] which represents a flywheel subjected to a torsional loading. The geometry of the flywheel is represented with a levelset that is interpolated on a level 9 octree mesh whose bounding box is $40 \%$ larger than the part's bounding box. The approximation mesh consists in a regular level 4 mesh (see figure 9(b)), but an adapted octree could also be considered with the use of a proper error estimator. The geometrical mesh is presented in figure 10(a), and the resulting integration cells in figure 10(b). The results are depicted in figure 11 for degree 4 and 7 X-FEM approximations (resp 4434 and 12978 dofs) and a conforming FEM degree 4 approximation (128 704 dofs, sub-parametric linear mesh). One can see that the stress distribution is consistent between FEM and X-FEM computations with much less degrees of freedom. If now the strain energy of the flywheel is computed, it can be seen that degree 4 X-FEM approximation leads to a $3.59 \%$ discrepancy for $3.45 \%$ of the dofs of the reference solution. Concerning the degree $7 \mathrm{X}$-FEM approximation, a $0.44 \%$ error is obtained with $10.1 \%$ of the dofs of the reference solution (see table 1 ).

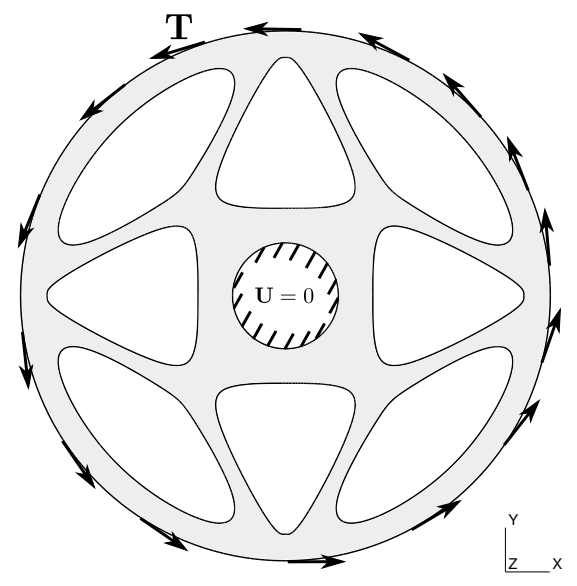

(a)

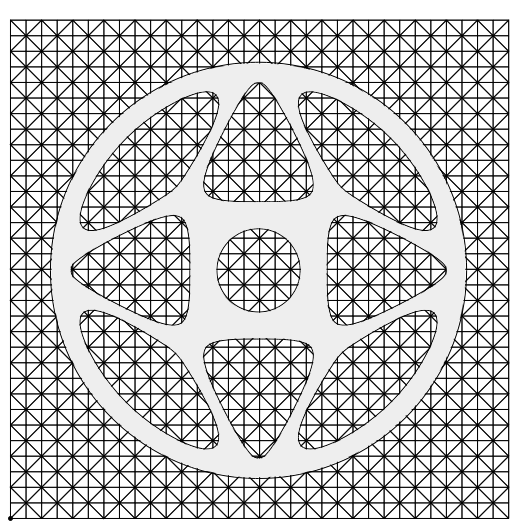

(b)

Figure 9: Flywheel subjected to a torsional loading: (a) Geometry and boundary conditions; (b) Approximation mesh. 


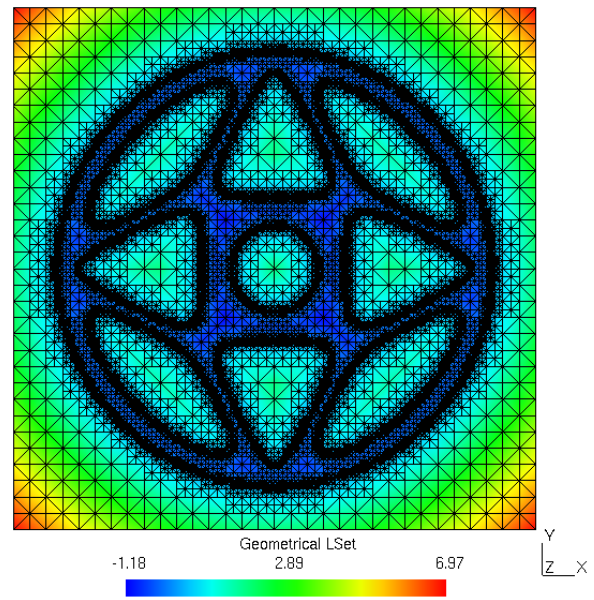

(a)

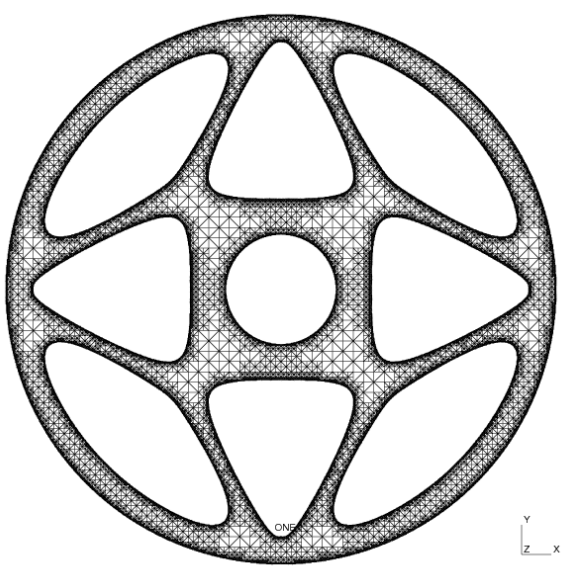

(b)

Figure 10: Flywheel subjected to a torsional loading: (a) Levelset on the geometrical octree; (b) Integration cells in the domain.

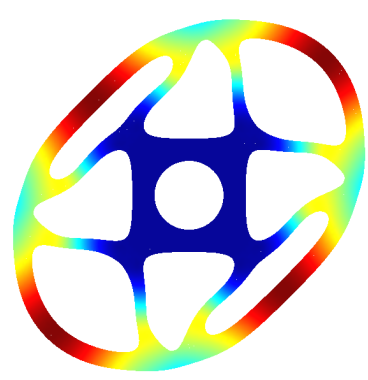

(a)

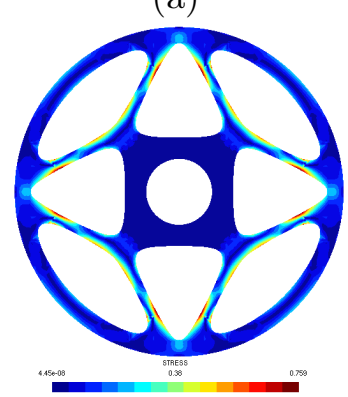

(c)

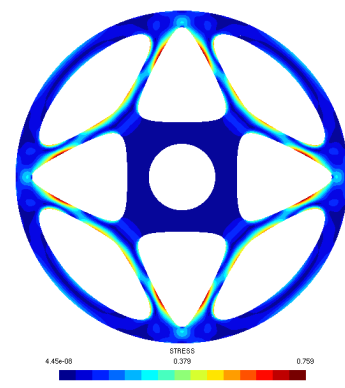

(b)

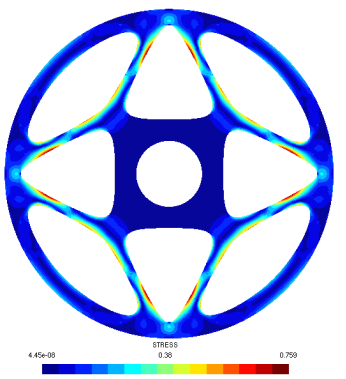

(d)

Figure 11: Flywheel subjected to a torsional loading: (a) Displacement, $p=4$ FEM approximation (64352 dofs); (b) Von Mises stress, $p=4$ FEM approximation; (c) Von Mises stress, $p=4$ X-FEM approximation (4434 dofs) and (d) Von Mises stress, $p=7$ X-FEM approximation (12 978 dofs) 


\begin{tabular}{ccc} 
Case & Nb Dofs (Ratio/Fem) & Error $/$ Fem \\
\hline \hline Fem & $128704(1.0)$ & Ref. \\
X-FEM $p=4$ & $4434(0.034451)$ & $3.5937 \%$ \\
X-FEM $p=7$ & $12978(0.10084)$ & $0.44273 \%$ \\
\hline
\end{tabular}

Table 1: Flywheel subjected to a torsional loading: Comparison between Fem and XFEM

\subsection{Free surfaces}

In this section, the accuracy of the approach is assessed in the case of free surfaces defined by means of level sets. In a first part, a simple model problem is studied. In a second one, complex geometries are considered.

\subsubsection{Influence of the geometrical accuracy}

Fixed geometrical accuracy The objective of this example is to compare the results obtained here with those already published in [25], in order to study the influence of the geometrical accuracy on both convergence rate and error level. Consider an infinite plate with a circular hole in its center in uniaxial tension $\sigma_{\infty}=1$. MPa along $\mathbf{x}$ axis (see figure 12). The analytical solution of this problem is given in [19] and is used here as a reference solution for error monitoring. Only a square of length $L=2 \mathrm{~mm}$ with a circular hole of radius $a=0.4 \mathrm{~mm}$ at its center is considered here. Finally, tractions computed from the exact solution are applied on the boundary of the domain, and three dofs are prescribed to zero in order to avoid rigid body motion. The exact solution of this finite problem is therefore the same as the exact solution inside the boundary. Young's modulus is set to 1.0 MPa, and Poisson's ratio to 0.3. A convergence study is performed, and the error in the energy norm is monitored with respect to the approximation mesh size. First, the case of a very fine geometrical mesh is considered: the size of the elements near the interface correspond to level 8 octree cells (see figure 13(a)). The elements of the approximation mesh correspond to cells of level 1 to 6 . The convergence for linear, quadratic and cubic approximations is depicted in figure 14(a). The order of convergence is optimal for linear and quadratic approximations on the whole extend of the curve whereas it begins to be degraded for the cubic approximation and very small $h$. This is related to fact that the geometrical accuracy becomes inadequate with respect to the approximation. However, the convergence rate is almost not degraded.

In a second time, the geometrical size is coarsened up to level 5 (see figure 13(b)). The convergence results from level 1 to level 5 for the approximation mesh are depicted in figure 14(b). One can see that in this case the geometrical accuracy is not sufficient on the whole extend of the curve for both quadratic and cubic approximations. However, note that the accuracy is still better with the cubic approximation. 


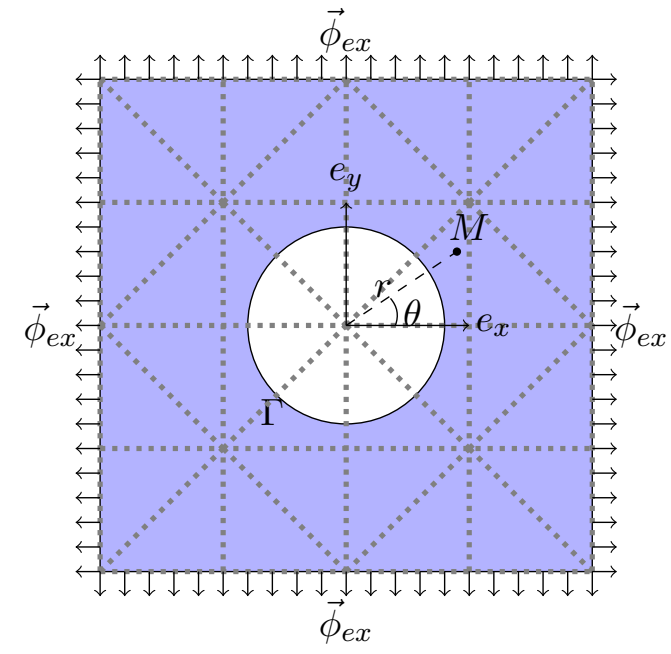

Figure 12: Infinite plate with a hole submitted to uniaxial tension: geometry and approximation mesh.

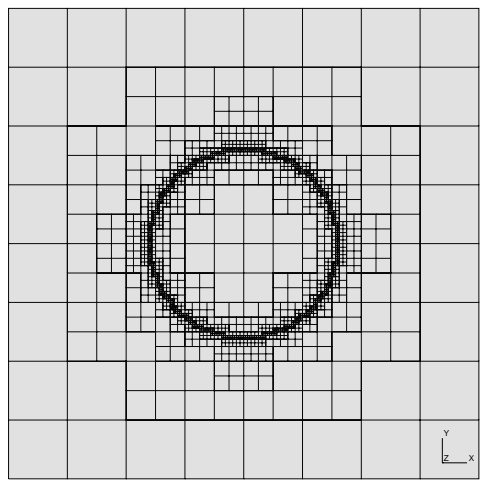

(a)

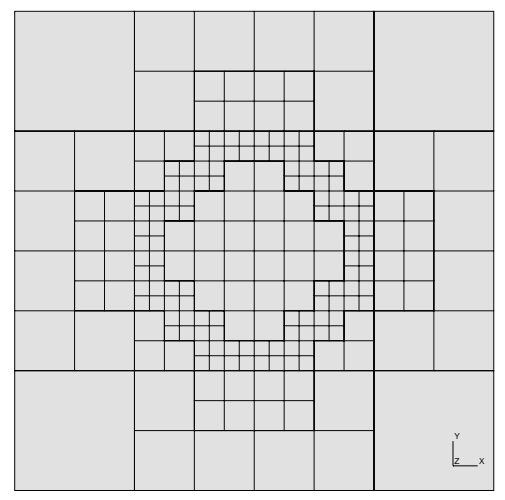

(b)

Figure 13: Geometrical octree: (a) fine case and (b) coarse case (the geometrical mesh is obtained by splitting the cells in two triangular elements). 


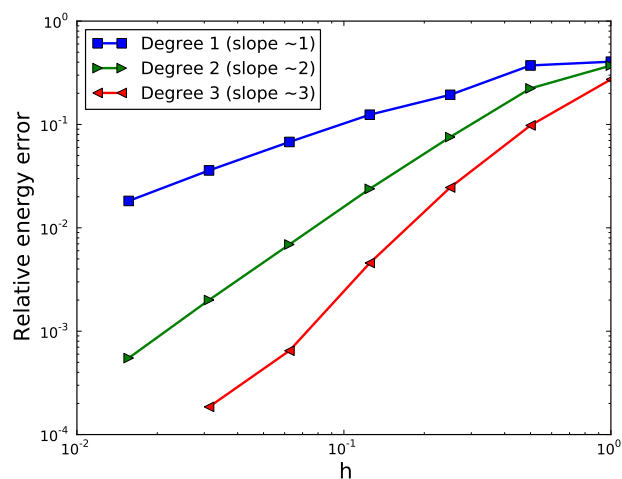

(a)

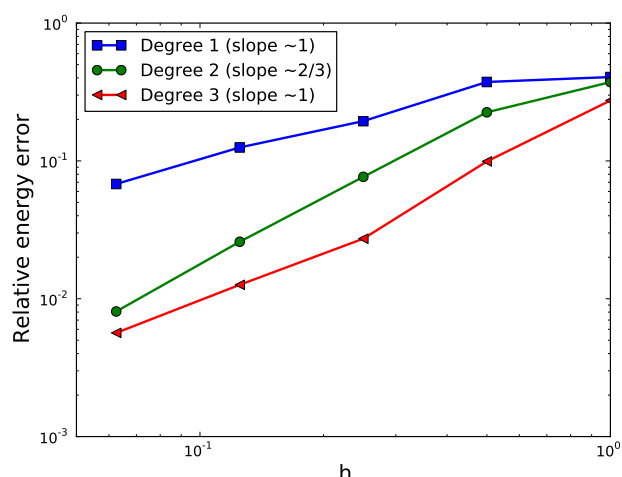

(b)

Figure 14: Case of a hole: Convergence with respect of $h$ for linear, quadratic and cubic approximations: (a) fine geometrical description, (b) coarse geometrical description. The exact slopes of the curves are given in Table 2 .

Optimal geometrical accuracy In a second step, the same example is treated, but the size of the geometrical mesh $h_{g}$ is obtained by means of the estimate proposed by Huerta et al. [38]:

$$
h_{g}=\alpha h^{2 p / 3}
$$

where $p$ is the order of the approximation. This estimate is based on the estimate of the approximation error of high order problems with linear boundaries [55]. It is applied in this case with $\alpha=1 / 30$. This value was obtained so that almost optimal convergence could be obtained for polynomial order 5 . As seen in figure 15, optimal asymptotic rate of converge is obtained for polynomial orders from 1 to 5 . Note that for quintic polynomials, the asymptotic slope could hardly be obtained because of the amount of degrees of freedom needed to obtain more points.

$p$-convergence Finally, the case of $p$ refinement is considered to assess the behaviour of the proposed approach. The same example is considered, and a uniform $p$ refinement is applied on a level 2 approximation mesh $(4 \times 4 \times 2$ triangular elements). The geometrical accuracy of the interface vary from level 10 to level 5 in order to study its influence on the convergence. The approximation space is defined by means of Lagrange polynomial functions from order 1 to order 9, and the results of the convergence study (error as a function of the number of degrees of freedom) are depicted in figure 16. It can be seen that an exponential convergence is obtained in all cases. Only the extend of this domain is influenced by the geometrical accuracy: The coarser the geometrical accuracy, the sooner the geometrical error degrades the convergence. However, $p$ refinement is more efficient than $h$ refinement. The Von-mises norm of the stress field corresponding to a $p=9$ approximation is depicted in figure 17: one can observe the high smoothness of this field, despite of a very coarse approximation mesh (32 triangles). 


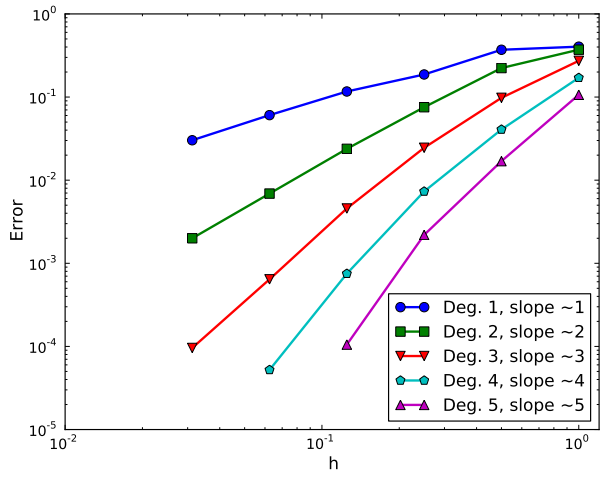

Figure 15: Case of a hole: convergence with respect to mesh size for $p=1$ to $p=5$. The exact slopes of the curves are given in Table 2

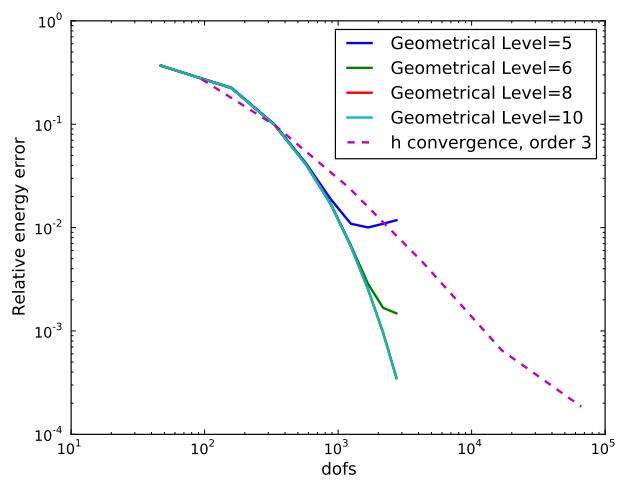

Figure 16: Case of a hole: Convergence with respect to the number of degree of freedom for a $p$ refinement and various geometrical accuracy. 


\begin{tabular}{ll} 
Figure, Order & Slope \\
\hline \hline $14(\mathrm{a}), \mathrm{P} 1$ & 0.92 \\
$14(\mathrm{a}), \mathrm{P} 2$ & 1.81 \\
$14(\mathrm{a}), \mathrm{P} 3$ & 2.62 then 1.81 \\
14 (b), P1 & 0.90 \\
$14(\mathrm{~b}), \mathrm{P} 2$ & 1.6 \\
$14(\mathrm{~b}), \mathrm{P} 3$ & 1.14 \\
\hline $15, \mathrm{P} 1$ & 0.97 \\
$15, \mathrm{P} 2$ & 1.79 \\
$15, \mathrm{P} 3$ & 2.79 \\
$15, \mathrm{P} 4$ & 3.85 \\
$15, \mathrm{P} 5$ & 3.29 then 4.38 \\
\hline
\end{tabular}

Table 2: Exact slopes for the infinite plate with a hole.

\subsubsection{Influence of the computational parameters}

Consider the example already considered in section 4.2.1. It was shown that exponential convergence could be obtained with a sufficient geometrical accuracy. We now investigate the influence of two parameters on the convergence properties of the proposed approach: (i) the geometrical accuracy and (ii) the size of the approximation elements with respect to the size of the hole. The numerical setup is similar to the one considered in section 4.2.1. First, the radius of the hole is decreased from $0.4 \mathrm{~mm}$ to $0.05 \mathrm{~mm}$ (keeping the same geometrical accuracy), and the convergence of the solution during a $p$ refinement is monitored in figure 18, curve A. It can be seen that the convergence is strongly degraded with respect to figure 16: The beginning of an exponential convergence is obtained, but a large number of dofs would be necessary to decrease the error level below $10^{-3}$. In addition, it can be seen that the efficiency of the $p$ refinement is hardly better than cubic $h$ one (see curve labeled "A, h conv"). This behaviour could stems from an insufficient geometrical accuracy, so that the same case is studied, using an improved geometrical representation (level 12 rather than level 9 mesh): It can be seen in curve B that this does not improve the behaviour, as the two curves match. Thus, we can conclude that the slow convergence of the solution is not related to the geometrical accuracy. The size of the approximation mesh is now decreased from level 4 to level 7. In this case, a clear exponential convergence is recovered, (see curve C) and a $10^{-4}$ error can be obtained at the price of more degrees of freedom (as the approximation mesh is finer). However, in this case $p$ order equal to 3 or 4 is sufficient to get this very small error level.

As a conclusion to this study, it has been shown that the size of the approximation elements should not be too large with respect to the size of the geometrical details in order to ensure a fast $p$-convergence of the approach. This can be explained by the fact that the length-scale of the perturbations caused by the hole is related to its size: when the radius of the hole is decreased from 0.4 to 0.05 , these perturbations becomes 


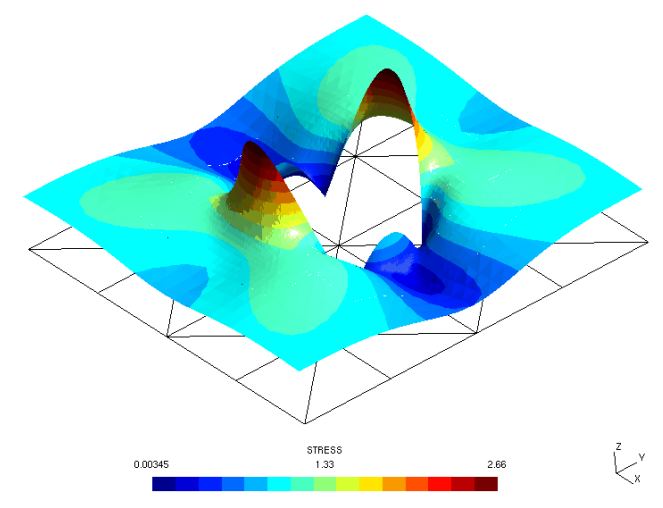

Figure 17: Case of a hole: Von-mises norm of the stress field, $p=9$. Note that in order to obtain this figure, the solution is plotted linearly on a mesh whose elements are obtained by recursively splitting those of the geometrical one. A more efficient adaptive alternative was proposed in [56]

high frequencies with respect to elements size, so that only very high order polynomials orders can capture it. Decreasing the size of the approximation mesh makes this influence appear as medium frequencies that are captures by moderate polynomial orders.

\subsubsection{Percolated domain}

The approach is now applied to the more complex example depicted in figure 19: a square plate of length $2.0 \mathrm{~mm}$ and drilled by 450 random holes of radius ranging from $2.10^{-3} \mathrm{~mm}$ to $10^{-1} \mathrm{~mm}$, and subjected to a $\mathbf{x}$ extension. The elements size of the approximation mesh is $0.125 \mathrm{~mm}$ (level 4), as depicted in figure 19. A reference solution is obtained by means of a level 10 mesh $\left(h=1.9510^{-3} \mathrm{~mm}\right.$ ) using X-FEM (linear approximation), and leading to 435297 dofs. The evolution of the deformation energy is studied when the polynomial order is increased, and the error with respect to the reference solution is plotted in figure 20. It can be seen that exponential converge is obtained and that less than $0.6 \%$ error can be achieved with $17 \%$ of the dofs needed by the reference solution. Note however that an extremely high order approximation was necessary to obtain this accuracy (degree 12). This behavior is related to the phenomenon highlighted in the last section. The displacement and stress fields associated to this polynomial order are presented in figure 21. The degree of smoothness of the stress field is highlighted.

\subsubsection{Application to a high resolution image}

The approach is now applied to image-based geometries. An integrated process leading to the levelsets describing the geometry was already presented in [40,41, 42, 43, 39], based on the ITK library [57]. As a high resolution image was not available, a model 


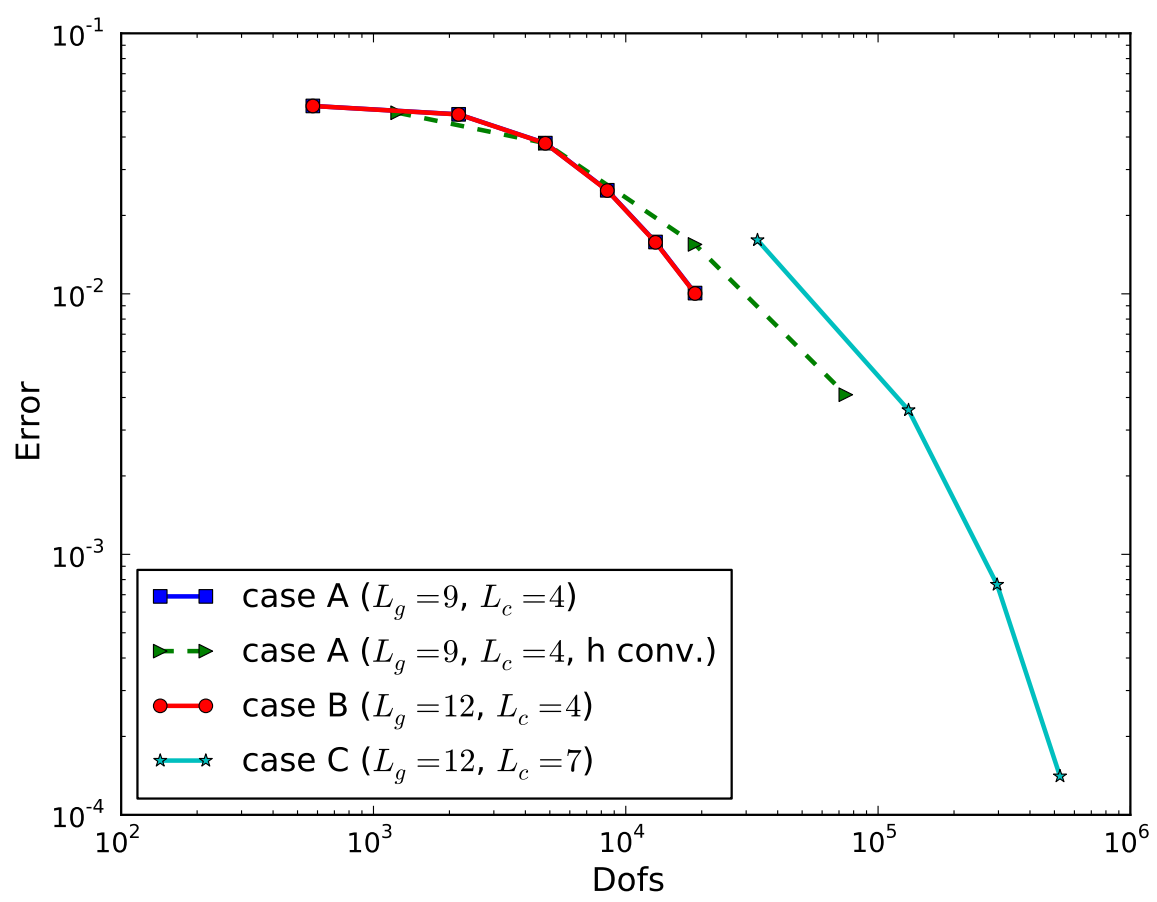

Figure 18: Influence of the geometrical accuracy and the size of the approximation elements.

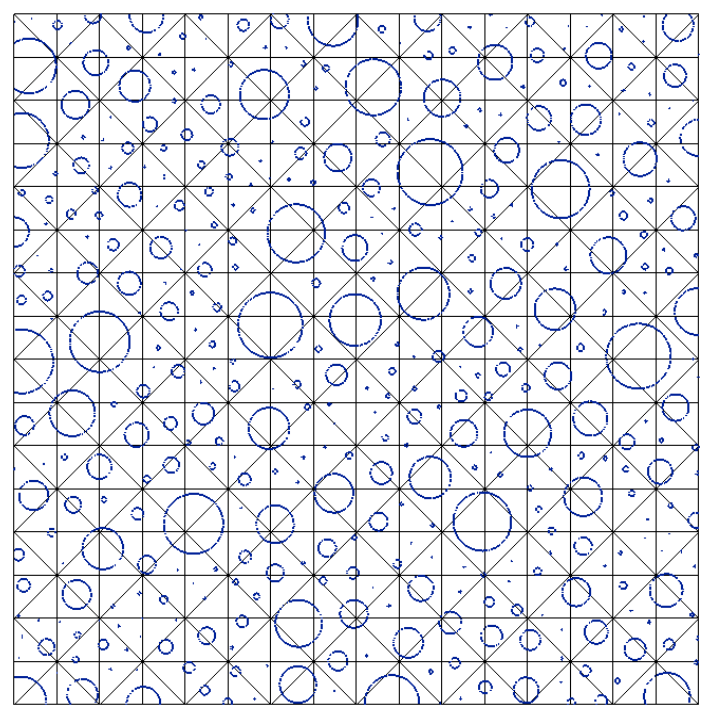

Figure 19: Percolated domain: Geometry and approximation mesh 


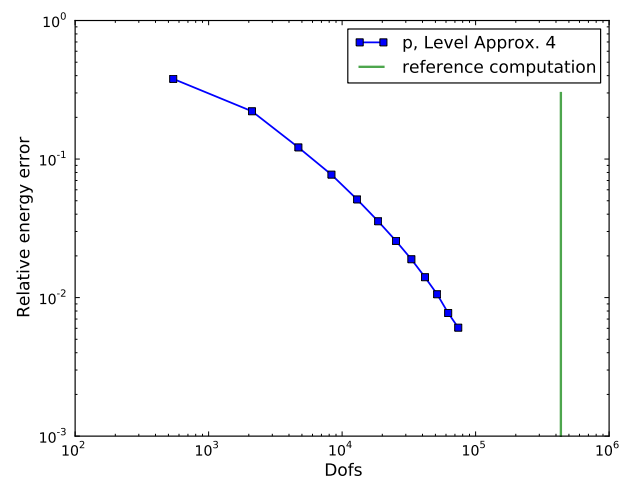

Figure 20: Percolated domain: $p$ convergence with respect to the number of degrees of freedom.

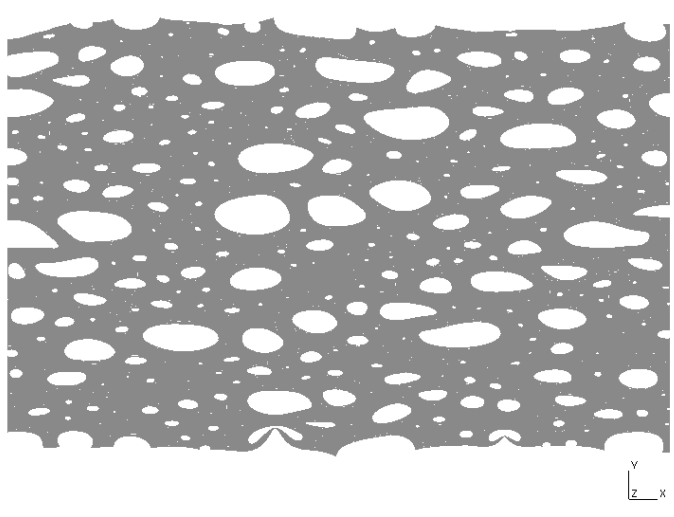

(a)

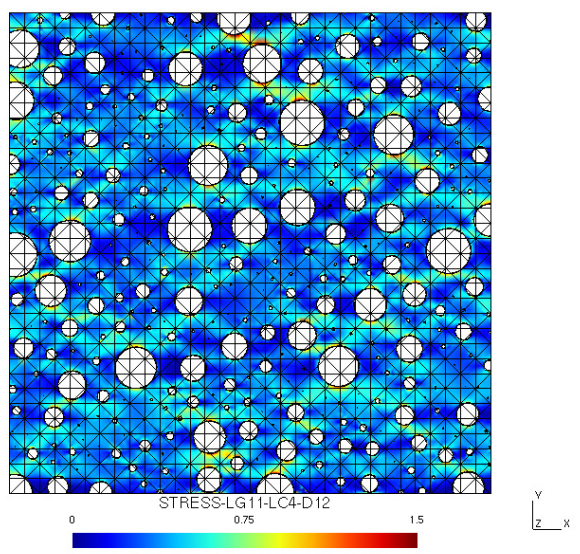

(b)

Figure 21: Percolated domain: (a) Deformed mesh ; (b) Von-Mises stress 
microstructure was generated by random insertion of circles in the computational domain. The objective here is to study the accuracy of the proposed approach for realistic geometries.

The geometry defined in figure $22(\mathrm{a})$ is considered here: It depicts a fiber-matrix composite, and the resolution of the input geometry is $2048 \times 2048$ pixels. Note that in this case, the fibers are considered as holes. The domain is subjected to a uniaxial traction along $\mathbf{y}$, by means of prescribed tractions on the upper and lower edges. Clearly, solving this problem by means of a pixel-based model would lead to a very costly numerical model. In contrast, a $32 \times 32 \times 2$ approximation mesh is considered here, whereas the geometrical one embed the full geometrical accuracy (the elements near the material interfaces have pixel-size). The discretized geometry and the approximation mesh are depicted in figure 22(a). A reference solution is obtained by means of a conforming quadratic sub-parametric finite element approximation defined on the mesh presented in figure 22(b) (the size of the elements are twice the size of the pixel here). A zoom on the geometry of the problem is also presented in figure 23: one can see that the shaded elements are cut three time by the iso-zero. Such a geometrical case could only be represented by a very high order levelset (quadratic or cubic is not sufficient), with all the difficulties that it implies (see Huerta et al. [38]). The solution corresponding to a degree 7 approximation (von-mises norm of the stress field, displacement field) is presented in figure 24: one can observe the smoothness of the stress field that is obtained.

The $p$ convergence of the solution is presented in figure 25 , and it is compared to $h$ convergence for linear, quadratic and cubic approximations.

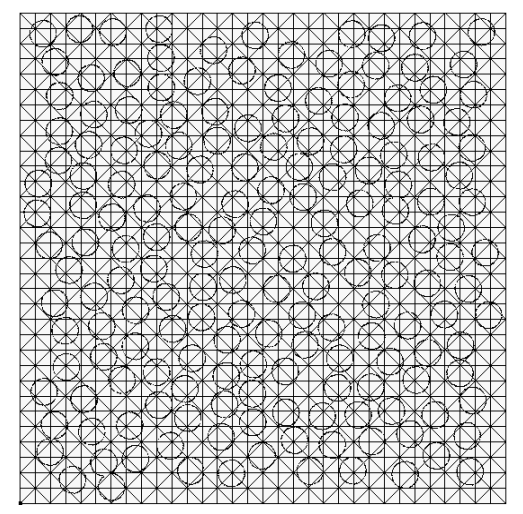

(a)

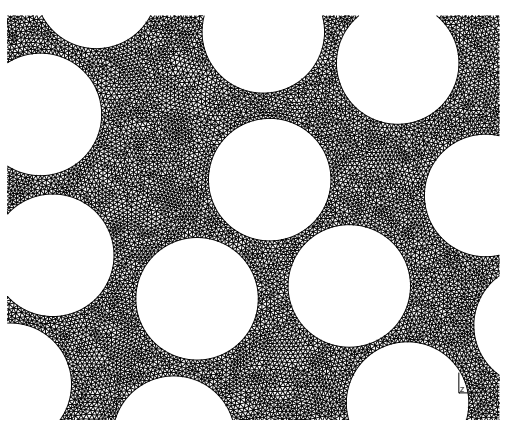

(b)

Figure 22: $2048 \times 2048$ pixels composite (hole): (a) Approximation mesh and material interfaces ; (b) Zoom on the reference finite element mesh

It can be seen that $p$ refinement is the most efficient approach: it outperforms largely linear and quadratic approximations, and slightly the cubic one. Higher polynomial degree should be necessary to emphasize the difference with cubic approximation. However, conditioning issues prevent the use of higher order approximations (greater than 7 here). This could be due to the use of Lagrange shape functions that are known to lead to ill 


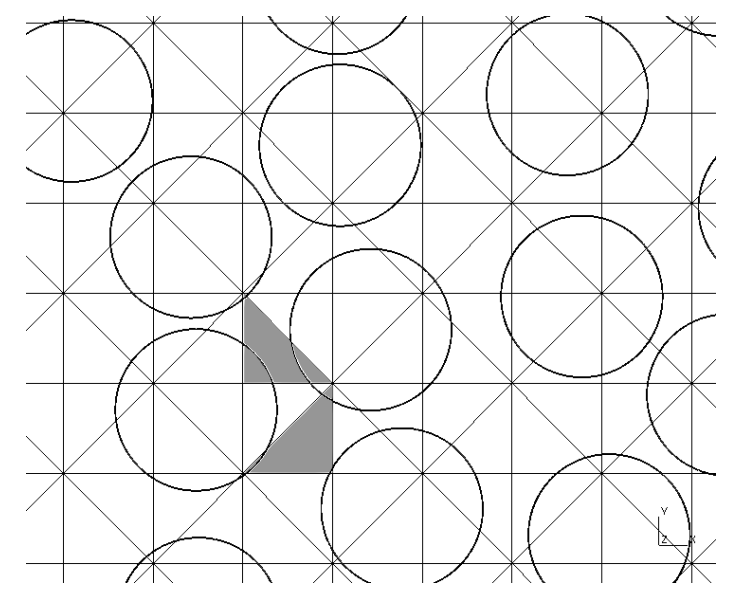

Figure 23: $2048 \times 2048$ pixels composite (hole): Zoom on the approximation mesh: The two elements in gray are cut three times by the iso-zero.

conditioned systems when polynomial order increases. The use of hierarchical shape functions would improve this limitation.

\subsection{Material Interfaces}

\subsubsection{Optimal Convergence}

The case of material interfaces is now considered, in order to assess the influence of the parameters of the approach, but also to study whether the Nitsche approach is a good candidate to recover the optimal convergence rate for high order approximations within $\mathrm{X}$-FEM. Consider an infinite plate subjected to equibiaxial tension and containing a circular inclusion of radius $a=0.4 \mathrm{~mm}$ at its center. The approximation domain consists in a square of length $2.0 \mathrm{~mm}$ centred on point $(0,0)$. The two materials are considered as linear elastic with Young's modulus $E_{m}=1.0 \mathrm{MPa}$ and Poisson's ratio $\nu_{m}=0.3$ for the matrix. Concerning the inclusion, the following parameters are considered: $E_{i}=$ $10.0 \mathrm{MPa}$ and $\nu_{i}=0.25$. The analytical solution of this problem was given in [19] and is used to apply exact tractions on the boundary of the approximation domain. First, a very fine geometrical mesh is considered: The size of the elements near the interface correspond to level 11 cells. The size of the elements of the approximation mesh vary from level 1 to level 7 cells. Nitsche's parameter $\beta$ is obtained by solving the eigenvalue problem (10) for $p=1,2,3$ and 4 , and $\alpha$ is set to $1 / 50$. This value was obtained so that optimal convergence could be obtained for polynomial order 4. As depicted in figure 26(a), convergence rates for both linear and quadratic approximations are optimal whereas the cubic approximation does not converge at the expected rate $\left(O\left(h^{3}\right)\right.$ in the energy norm). This is due to an insufficient geometrical representation of the interface. However the error level is significantly lower than the quadratic one. If now the size of the geometrical octree is set according to the estimate proposed by Huerta et al. 


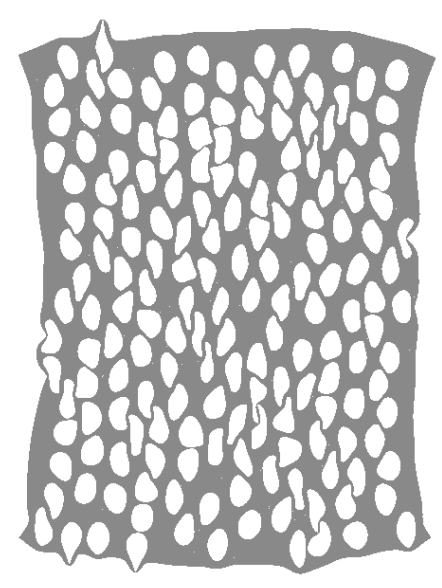

(a)

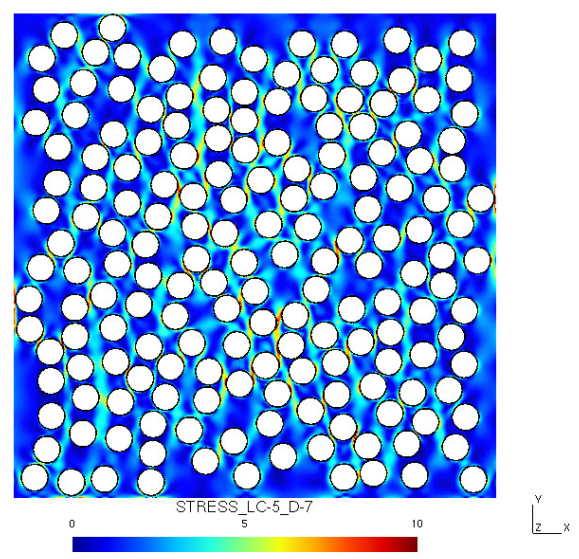

(b)

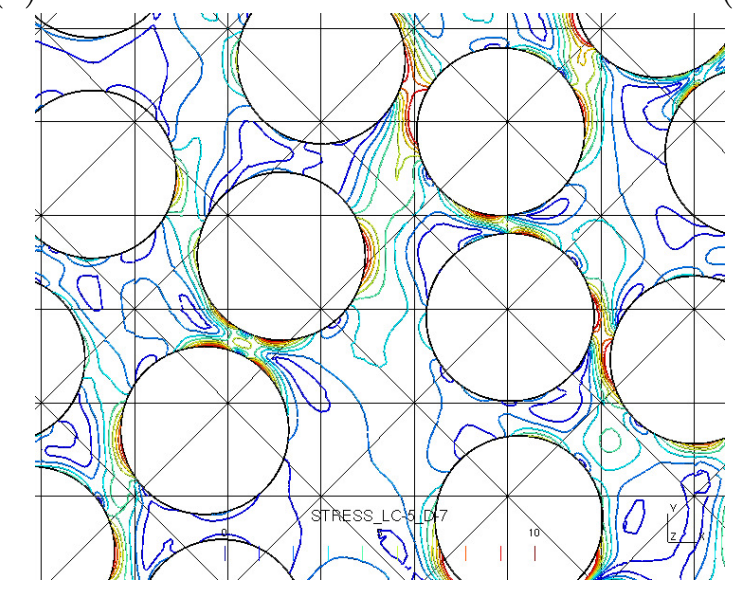

(c)

Figure 24: $2048 \times 2048$ pixels composite (hole): (a) Deformed configuration ; (b) Vonmises norm of the stress field $(p=7)$; (c) iso-values of the von-mises norm of the stress field ( $p=7$, zoom). 


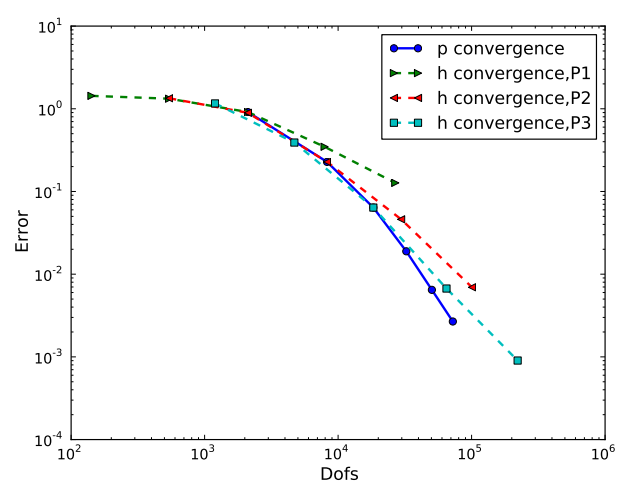

Figure 25: $2048 \times 2048$ pixels composite (hole): Convergence with respect to the number of degrees of freedom.

[38], optimal convergence rates can be recovered, as depicted in figure 26(b). This shows that provided that the geometrical accuracy is sufficient, the approach proposed here (and thus the one proposed in [25]) allows to construct optimal high order XFEM approximations in the case of material interfaces. The case of $\mathrm{p}$ convergence is now considered: An approximation mesh composed of level 2 elements is considered $(2 \times 2 \times 2$ triangular elements), and a geometrical mesh with refined elements along the interface are considered. Four geometrical meshes are considered depending on the size of the elements near the interface (level 5, 6, 8 and 10). In all the cases, the value of Nitsche's parameter is obtained by solving the eigenvalue problem associated to the stability condition. The results are depicted in figure 27. The results are similar to the one obtained for holes: An exponential convergence is obtained during $p$ refinement provided that the geometrical accuracy is sufficient. When it is no more the case, the convergence slows down, then stops at a level that depends on the geometrical accuracy. It is worth noting that $p$ refinement is slightly less effective in the case of material interfaces than in the case of holes. Indeed, it can be seen that the improvement in the accuracy with $p$ refinement is less obvious than in the case of holes, and also that the influence of the geometrical accuracy is more pronounced. This behavior is partially linked to the contrast between the inclusion and the matrix. A comparison between contrast 10 and 100 is presented in figure 27 (with the same geometrical accuracy of level 10). It can be seen that the contrast has a limited influence on the range of optimal convergence, which means that estimate (13) is hardly influenced by the contrast (at least, in this case). On the contrary, the level of the plateau is significantly influenced by the contrast level.

\subsubsection{Application to a high resolution image}

Consider the composite material already presented in section 4.2.4. The resolution of the image defining the problem is $2048 \times 2048$ pixels. The matrix is composed of a linear 


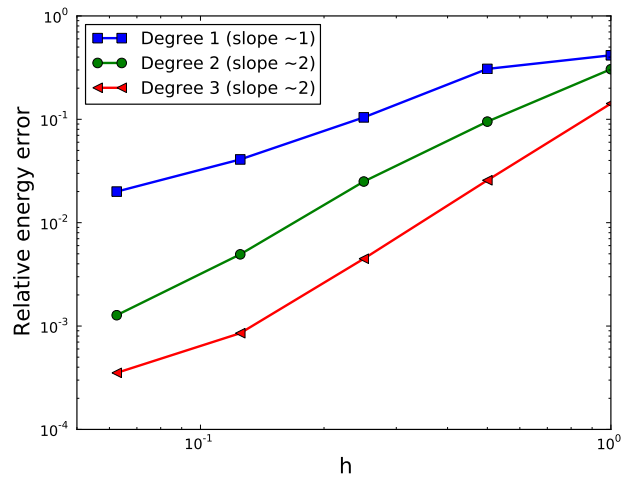

(a)

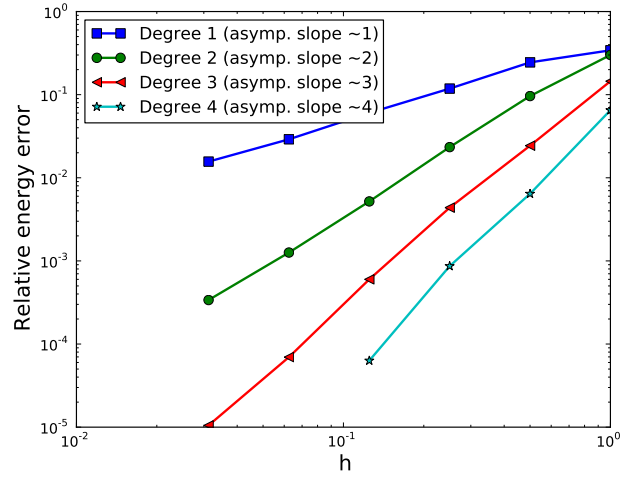

(b)

Figure 26: Case of material interfaces: Convergence with respect to $h$ for linear, quadratic and cubic approximations. (a) fine geometrical description and (b) following [38] for the geometrical description. The exact slopes of the curves are given in Table 3

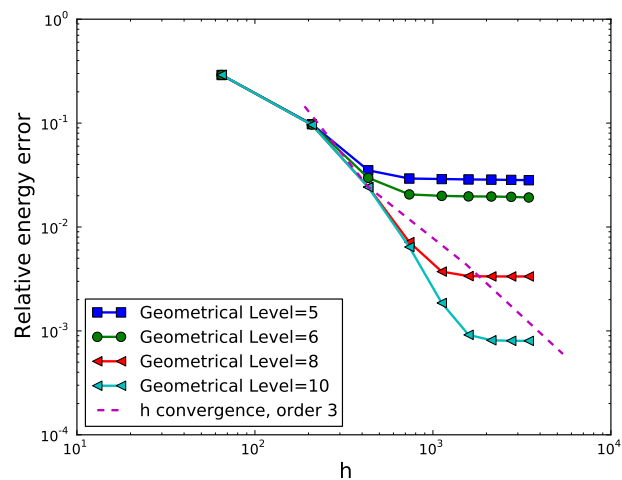

(a)

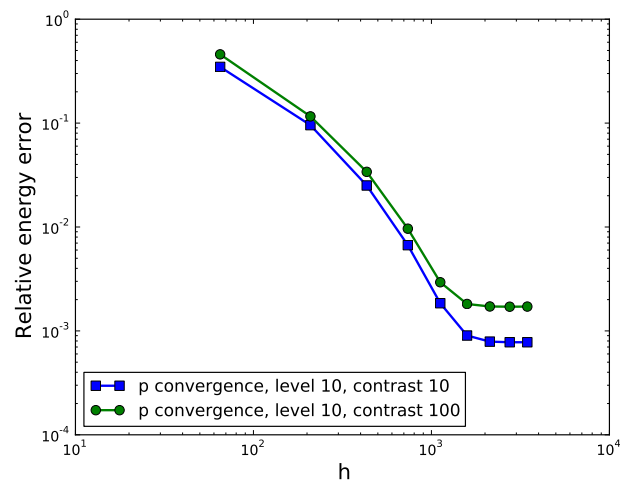

(b)

Figure 27: Case of material interfaces: $p$ Convergence with respect to the number of dofs. (a) influence of the geometrical accuracy; (b) influence of the contrast. (Note that the geometrical description of the cubic $h$ convergence was obtained following [38]) 


\begin{tabular}{ll} 
Figure, Order & Slope \\
\hline \hline 26 (a), P1 & 0.99 \\
$26(\mathrm{a}), \mathrm{P} 2$ & 2.01 \\
$26(\mathrm{a}), \mathrm{P} 3$ & 2.47 then 1.27 \\
26 (b), P1 & 1.0 \\
$26(\mathrm{~b}), \mathrm{P} 2$ & 2.05 \\
$26(\mathrm{~b}), \mathrm{P} 3$ & 2.92 \\
$26(\mathrm{~b}), \mathrm{P} 4$ & 3.34 then 3.78 \\
\hline
\end{tabular}

Table 3: Exact slopes for the infinite plate with an inclusion.

elastic material with Young's modulus $E=1.0 \mathrm{MPa}$ and Poisson's ration $\nu=0.3$. The fibers are made of a linear elastic material with Young's modulus $E=10.0 \mathrm{MPa}$ and Poisson's ration $\nu=0.25$. The material sample is subjected to a uniaxial tension along $\mathbf{y}$ axis by means of uniform unit tractions along the upper and bottom boundaries of the domain. A conforming mesh is built in order to obtain a reference solution. This mesh is presented in figure 28 and contains 228940 quadratic triangles, which leads to 584555 dofs. The energy computed with this mesh is compared with the one obtained with the proposed approach. A level 5 approximation mesh is considered $(32 \times 32 \times 2$ triangular elements) in addition with an adapted geometrical mesh (pixel-size near the interfaces, and as coarse as possible away from the interface). Two set of convergence studies are considered in the following:

- $h$ convergence for $p=1,2$ and 3 using approximation meshes from level $3(8 \times 8 \times 2$ elements) to level $7(128 \times 128 \times 2$ elements $)$;

- $p$ convergence for $p=1$ to $p=8$ using a level $5(32 \times 32 \times 2$ elements $)$ approximation mesh.

In the first case, Nitsche's parameter is computed using the eigenvalue problem, whereas it is set to 4000 for $p \geq 4$ in the second case. This last value was obtained by extrapolation of the values considered for $p=1,2$ and 3 (this choice was made as in this case the size of the eigenvalue problem is close to the size of the whole mechanical problem). Considering the local form of the eigenvalue problem would decrease the cost of the procedure. Note that, as advocated by Tran et al. [51], multiple enrichments are considered in the approximation (see section 3.4). Without this approach, the convergence is strongly degraded.

The results of the convergence study are presented in figure 29: Both three solutions converge to the reference one. Using quadratic approximation doubles the rate of convergence with respect to linear approximation. Cubic approximation does not improve the convergence rate, but it is still more efficient than second order approximation as the constant in the error estimate is significantly improved: A $0.5 \%$ error is obtained with $80 \%$ less dofs than the reference solution. The case of $p$ convergence is not presented, as conditioning issues prevented us to use polynomial orders above 4 . This may 
be related to the fact that Lagrange shape functions were considered: it is known that these shape functions lead to ill-conditioned stiffness matrices. Moreover, each shape function is enriched several times with Heaviside functions which degrades even more the conditioning number. In addition, Nitsche's parameter may not be optimal in this case. However, even the cubic approximation is very efficient as it leads to a $3.1 \%$ error with respect to the reference solution by using $93 \%$ less dofs than the reference solution.

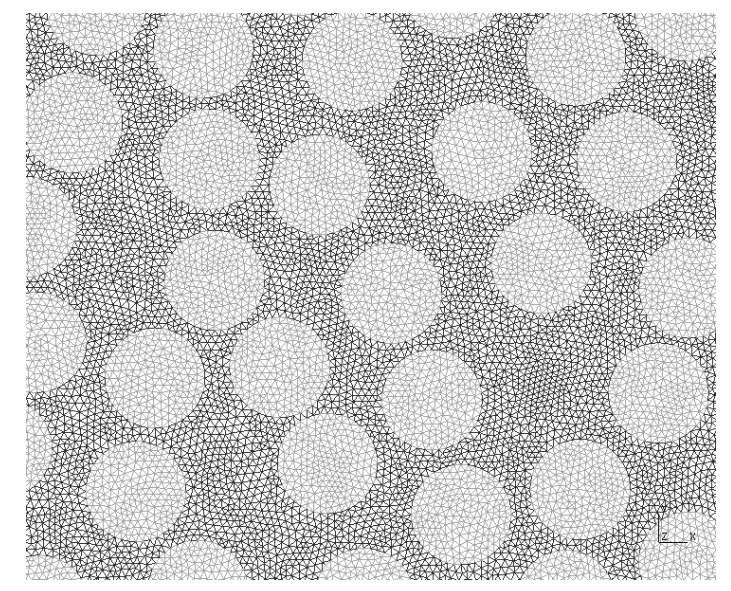

Figure 28: Zoom on the conforming finite element mesh.

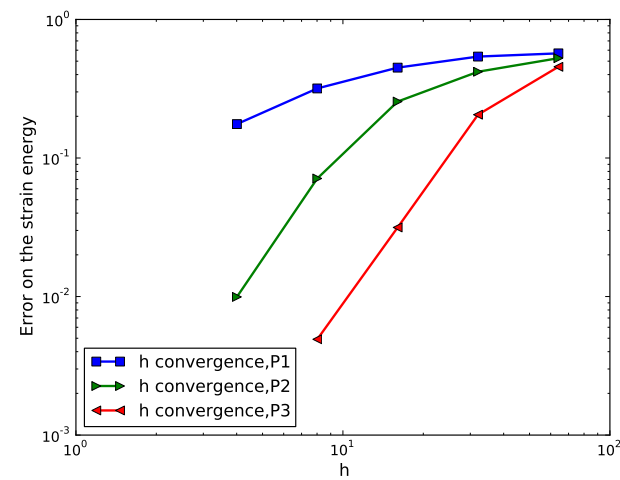

(a)

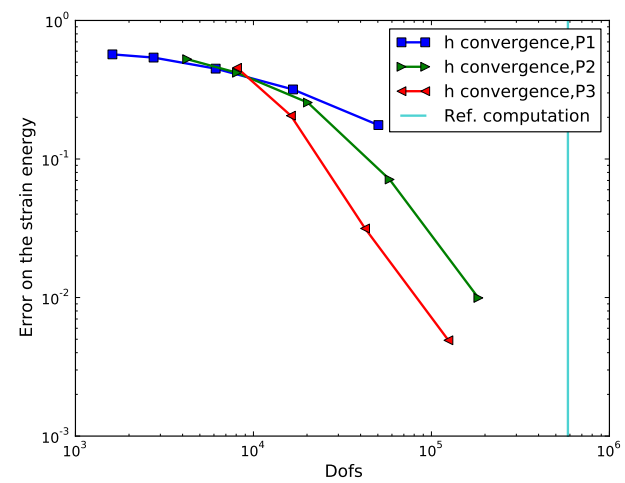

(b)

Figure 29: $2048 \times 2048$ pixels composite, $h$ refinement: (a) Error on the strain energy versus $h$; (b) Error on the strain energy versus number of dofs (Reference: 584555 dofs) 


\subsection{Numerical Homogenization}

The approach is finally applied to the numerical homogenization of image-based microstructures. The micro-structure considered here is the composite material defined in the last section, although we are now interested in the effective properties of this material. The effective elasticity tensor $\mathcal{C}_{\text {eff }}$ is looked, by means of the geometry of the micro-structure and the elasticity tensor of the two phases $\mathcal{C}_{\text {inclusion }}$ and $\mathcal{C}_{\text {matrix }}$. This tensor relates the macroscopic stress and strain tensors, $\underline{\underline{\Sigma}}$ and $\underline{\underline{E}}$ :

$$
\underline{\underline{\Sigma}}=\mathcal{C}_{e f f} \underline{\underline{E}}
$$

where $\underline{\underline{\underline{\Sigma}}}$ and $\underline{\underline{E}}$ are defined by the following spatial averages:

$$
\begin{aligned}
\underline{\underline{\underline{E}}} & =\langle\underline{\underline{\sigma}}\rangle \\
\underline{\underline{E}} & =\langle\underline{\underline{\varepsilon}}\rangle \\
<\bullet> & =\frac{1}{V} \int_{\Omega} \bullet d \Omega
\end{aligned}
$$

where $\underline{\sigma}$ and $\underline{\varepsilon}$ are the micro stress and strain tensors. The homogenization procedure is based on kinematic uniform boundary conditions (KUBC), but could also be based on stress uniform boundary conditions (SUBC) or periodic boundary conditions (PBC) $[58,59,60]$ :

$$
\mathbf{u}=\underline{\underline{E}} \cdot \mathbf{x} \quad \text { on } \partial \Omega
$$

Using these boundary conditions, equation (16) is automatically verified, whereas the terms of $\mathcal{C}_{\text {eff }}$ are obtained by computing eq.(15) for three macroscopic displacement modes $\underline{\underline{E}}_{1}, \underline{\underline{E}}_{2}$ and $\underline{\underline{E}}_{3}$ (macroscopic tractions and shear):

$$
\begin{aligned}
& \underline{\underline{E}}_{1}=\left[\begin{array}{ll}
1 & 0 \\
0 & 0
\end{array}\right] \\
& \underline{\underline{E}}_{2}=\left[\begin{array}{ll}
0 & 0 \\
0 & 1
\end{array}\right] \\
& \underline{\underline{E}}_{3}=\left[\begin{array}{cc}
0 & 0.5 \\
0.5 & 0
\end{array}\right]
\end{aligned}
$$

As in the last section, a conforming finite element model is considered as the reference solution. This reference solution is compared to the solution obtained with a conforming mesh with elements whose size is two times the pixel-size. The X-FEM solution is computed on a $32 \times 32 \times 2$ mesh with approximations from $p=1$ to $p=4$. In such an application, the extra computations that are needed for the approach are only done as a preprocessing step, then reused three times for the determination of the effective properties. In addition, the size of the three problems are smaller than in the case of conforming finite elements. The micro-stress associated to $\mathrm{x}$ uniaxial traction are presented in figure 30. Finally, the homogenized properties obtained for the composite material are presented in figure 31. It can be seen that the effective properties converges to the reference one, and that an accuracy below $3 \%$ can be obtained with $73 \%$ less dofs. 


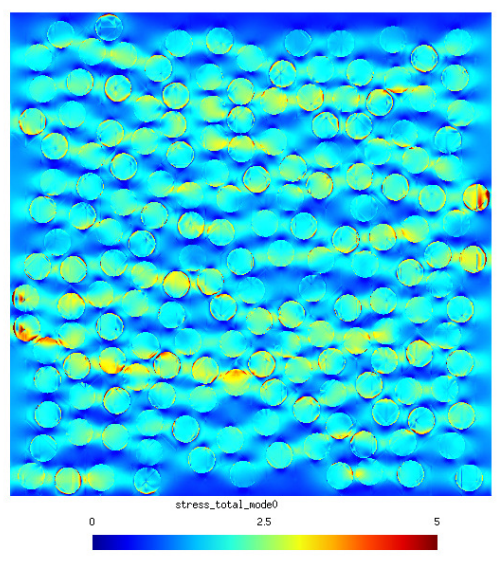

Figure 30: Micro stress field in the case of a Macro elongation along $\mathbf{x}$ axis for the composite material: $p=4$ approximation.

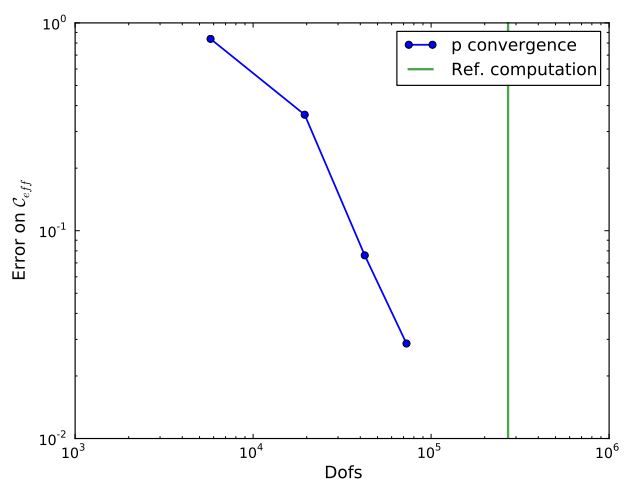

Figure 31: Convergence of the homogenized properties with respect to the number of degrees of freedom (composite material). $32 \times 32 \times 2$ mesh, $p=1 . .4$. 


\subsection{D example}

The proposed approach is finally applied to a 3D example. Consider the geometry depicted in figure 32: it is the segmented geometry obtained from a bone CT scan following the procedure proposed in [39]. This raw CT scan is available on the Internet through the BEL Repository managed by the Istituti Ortopedici Rizzoli, Bologna, Italy. The objective is to apply the proposed approach in order to compute the effective properties of this microstructure. The material is considered as linear-elastic with unit Young's modulus and Poisson's ration equal to 0.3. The resolution of the volume is $129^{3}$ voxels, which corresponds to a level 8 octree. The computational mesh is chosen as a level 5 octree $(32 \times 32 \times 32 \times 6$ tetrahedral elements). The evolution of the effective stiffness along $\mathbf{x}$ is monitored with polynomial order increased from 1 to 5 (37665 to 3691215 dofs). This quantity is evaluated following the approach detailed in the last section with KUBC boundary conditions. The convergence of the apparent modulus is monitored with respect to the number of dofs and compared to the asymptotic extension of the linear solution in figure 34. The reference solution was obtained by means of the extrapolation technique proposed in [53]. It can be seen that the behaviour of the high-order approach is far better than the one of the linear solution. Note however that only the pre-exponential range could be obtained with quintic polynomial order. Nevertheless, the efficiency of the approach is obvious.

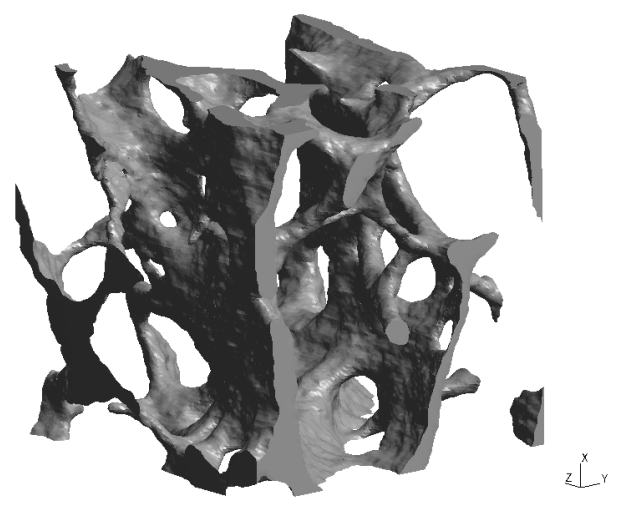

Figure 32: Segmented 3D bone microstructure. The raw CT scan is available on the Internet through the BEL Repository managed by the Istituti Ortopedici Rizzoli, Bologna, Italy.

\section{Conclusion}

In this paper, a technique was presented in order to uncouple geometrical description and approximation in the context of the X-FEM plus levelset approach. Earlier attempts have been made on this subject in the context of the generalized finite element method (G-FEM) $[18,12]$, but in the case where the interface is represented explicitly. Here, an 


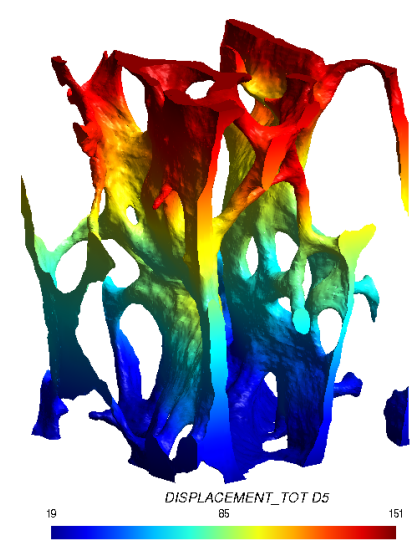

(a)

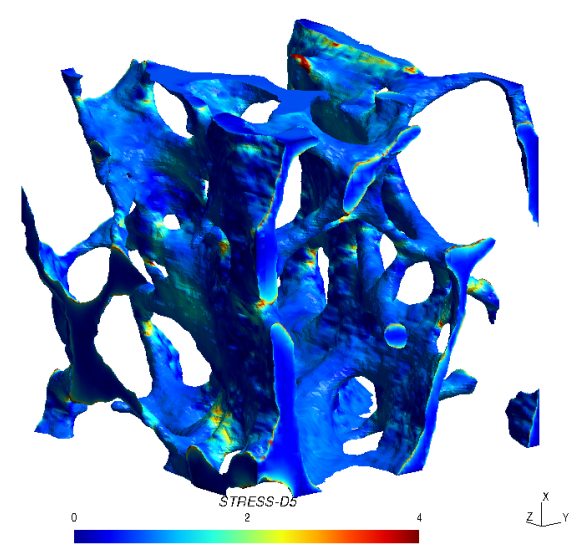

(b)

Figure 33: 3D bone microstructure. (a) Total displacement from elongation mode along $\mathbf{x}(p=5) ;(\mathrm{b})$ Micro-Stress field $(p=5)$

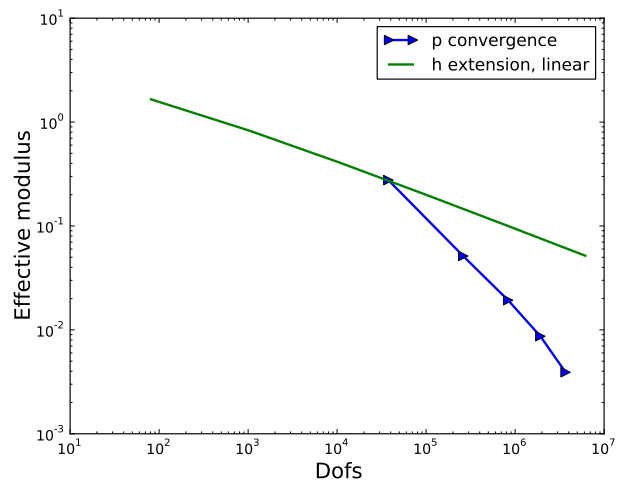

Figure 34: Convergence of the effective stiffness modulus along $\mathbf{x}$. Comparison between $p$ refinement and $h$ extension. 
implicit representation based on the levelset approach was used. From a full resolution model, two meshes are built: (i) a regular and coarse approximation mesh on which the interpolations of the mechanical fields are defined (of arbitrary order) and (ii) an adapted quadtree mesh that preserves the geometrical accuracy of the interfaces. As these two meshes are built from the same quadtree structure, the father of each geometrical element is known directly. This geometrical mesh is used as a sub-mesh for the approximation elements that are enriched by means of the X-FEM, as proposed by Dréau et al. [25] in the context of high- order X-FEM. We follow the same approach in order to improve the accuracy of the approach for coarse approximation meshes. In addition, this approach allows to represent interface shapes that could not be represented with high order (2 or 3 ) levelsets, as proposed in [26]. The use of Nitsche's approach was also considered in order to treat the case of material interfaces. It was shown that optimal convergence rates could be recovered provided that the geometrical mesh is sufficiently fine, which is the case when following the estimate proposed by Huerta et al. [38]. Then, the approach was applied for the simulation of image-based micro-structures in both 2D and 3D (direct calculation and homogenization). It was shown that $p$ refinement was very efficient in the case of free surfaces. For material interfaces, conditioning becomes an issue which prevent the use of very high order polynomials. However, even quadratic and cubic approximations leads to a visible improvement for a fixed number of degrees of freedom. It was also demonstrated that the approach give accurate results in the context of micromechanical homogenization with a low number of dofs. Work is currently in progress to continue the extension of this approach to the $3 \mathrm{D}$ setting for the homogenization of image-based micro-structures. It was finally shown how the approach could be used to define high order fictitious domain approaches with an accurate representation of the geometry. Note also that the approach could also be considered for the propagation of smooth curved cracks, using a scheme similar to the one proposed by Prabel et al. [27] or even improve it with a high-order X-FEM approximation as demonstrated in [61].

\section{References}

[1] P. Frey and P. George. Mesh Generation: Application to Finite Elements. Hermes Science Publishing Ltd;, 2000.

[2] Matt Staten. Why is hex meshing so hard?, what is the cubit team doing about it? In Cubit User's Meeting, 2007.

[3] V.K. Saulèv. On the solution of some boundary value problems on high performance computers by fictitious domain method. Siberian Math. J., 4:912-925, 1963.

[4] S. Del Pino and O. Pironneau. A fictitious domain based general pde solver. In Y. Kuznetsov, P. Neittanmaki, and O. Pironneau, editors, Numerical Methods for Scientific Computing Variational Problems and Applications. CINME, Barcelona, 2003. 
[5] V. Girault and R. Glowinski. Error analysis of a fictitious domain method applied to a dirichlet problem. Japan Journal of Industrial and Applied Mathematics, 12:487$514,1995$.

[6] A. Hansbo, P. Hansbo, and MG. Larson. A finite element method on composite grids based on nitsche's method. ESAIM: Mathematical Modelling and Numerical Analysis, 37:495-514, 2003.

[7] Jamshid Parvizian, Alexander Duster, and Ernst Rank. Finite cell method - $\mathrm{h}$ and p extension for embedded domain problems in solid mechanics. Computational Mechanics, 41(1):121-133, 2007.

[8] A Duster, J Parvizian, Z Yang, and E Rank. The finite cell method for threedimensional problems of solid mechanics. Computer Methods in Applied Mechanics and Engineering, 197(45-48):3768-3782, 2008.

[9] D. Schillinger, A. Düster, and E. Rank. The hp-d-adaptive finite cell method for geometrically nonlinear problems of solid mechanics. International Journal for $\mathrm{Nu}$ merical Methods in Engineering, pages n/a-n/a, 2011.

[10] Dominik Schillinger and Ernst Rank. An unfitted hp-adaptive finite element method based on hierarchical b-splines for interface problems of complex geometry. Computer Methods in Applied Mechanics and Engineering, 200(47-48):3358 - 3380, 2011.

[11] Vinh Phu Nguyen, Timon Rabczuk, Stéphane Bordas, and Marc Duflot. Meshless methods: A review and computer implementation aspects. Mathematics and Computers in Simulation, 79:763-813, 2008.

[12] T. Strouboulis, I. Babuška, and K. Copps. The design and analysis of the generalized finite element method. Computer Methods in Applied Mechanics and Engineering, 181:43-69, 2000.

[13] C. A. Duarte, I. Babuška, and J.T. Oden. Generalized finite element methods for three-dimensional structural mechanics problems. Computers and Structures, $77(2): 215-232,2000$.

[14] N. Moës, J. Dolbow, and T. Belytschko. A finite element method for crack growth without remeshing. International Journal for Numerical Methods in Engineering, 46:131-150, 1999.

[15] S. Osher and J.A. Sethian. Fronts propagations with curvature dependent speed: Algorithms based on Hamilton-Jacobi formulations. Journal of Computational Physics, 79:12-49, 1988.

[16] N. Moës, A. Gravouil, and T. Belytschko. Non-planar 3D crack growth by the extended finite element and level sets. Part I: Mechanical model. International Journal for Numerical Methods in Engineering, 53:2549-2568, 2002. 
[17] A. Gravouil, N. Moës, and T. Belytschko. Non-planar 3D crack growth by the extended finite element and level sets. Part II: level set update. International Journal for Numerical Methods in Engineering, 53:2569-2586, 2002.

[18] J. P. Pereira, C. A. Duarte, D. Guoy, and X. Jiao. hp -Generalized FEM and crack surface representation for non-planar 3-D cracks. International Journal for Numerical Methods in Engineering, 77(5):601-633, 2009.

[19] N. Sukumar, D. L. Chopp, N. Moës, and T. Belytschko. Modeling Holes and Inclusions by Level Sets in the Extended Finite Element Method. Comp. Meth. in Applied Mech. and Engrg., 190:6183-6200, 2001.

[20] N. Moës, M. Cloirec, P. Cartraud, and J.-F. Remacle. A computational approach to handle complex microstructure geometries. Comp. Meth. in Applied Mech. and Engrg., 192:3163-3177, 2003.

[21] Alejandro M. Aragón, C. Armando Duarte, and Philippe H. Geubelle. Generalized finite element enrichment functions for discontinuous gradient fields. International Journal for Numerical Methods in Engineering, 82(2):242-268, 2010.

[22] T. Strouboulis, L. Zhang, and I. Babuška. Generalized finite element method using mesh-based handbooks: application to problems in domains with many voids. Computer Methods in Applied Mechanics and Engineering, 192(28-30):3109-3161, 2003.

[23] Thomas-Peter Fries and Ted Belytschko. The extended/generalized finite element method: An overview of the method and its applications. International Journal for Numerical Methods in Engineering, 84(3):253-304, 2010.

[24] Abdelaziz Yazid, Nabbou Abdelkader, and Hamouine Abdelmadjid. A state-of-theart review of the x-fem for computational fracture mechanics. Applied Mathematical Modelling, 33(12):4269 - 4282, 2009.

[25] Kristell Dréau, Nicolas Chevaugeon, and Nicolas Moës. Studied X-FEM enrichment to handle material interfaces with higher order finite element. Computer Methods in Applied Mechanics and Engineering, 199(29-32):1922-1936, 2010.

[26] Kwok Wah Cheng and Thomas-Peter Fries. Higher-order XFEM for curved strong and weak discontinuities. International Journal for Numerical Methods in Engineering, 82:564-590, 2009.

[27] B. Prabel, A. Combescure, A. Gravouil, and S. Marie. Level set X-FEM nonmatching meshes: application to dynamic crack propagation in elastic-plastic media. International Journal for Numerical Methods in Engineering, 69(8):1553-1569, 2007.

[28] J. Rannou, N. Limodin, J. Réthoré, A. Gravouil, W. Ludwig, M.C. BaïettoDubourg, J.Y. Buffière, A. Combescure, F. Hild, and S. Roux. Three dimensional 
experimental and numerical multiscale analysis of a fatigue crack. Computer Methods in Applied Mechanics and Engineering, 199(21-22):1307-1325, 2010.

[29] J. Nitsche. Über ein Variationprinzip zur lösung von Dirichlet-Problem bei Verwendung von Teilräumen, die keinen Randbedingungen unterworfen sind. Abh. Math. Sem. Univ. Hamburg, 36:9-15, 1971.

[30] Anita Hansbo and Peter Hansbo. A finite element method for the simulation of strong and weak discontinuities in solid mechanics. Computer Methods in Applied Mechanics and Engineering, 193(33-35):3523-3540, August 2004.

[31] John Dolbow and Isaac Harari. An efficient finite element method for embedded interface problems. International Journal for Numerical Methods in Engineering, 78(2):229-252, 2009.

[32] Anand Embar, John Dolbow, and Isaac Harari. Imposing dirichlet boundary conditions with nitsche's method and spline-based finite elements. International Journal for Numerical Methods in Engineering, 83:877-898, 2010.

[33] J.M. Melenk and I. Babuška. The partition of unity finite element method : Basic theory and applications. Comp. Meth. in Applied Mech. and Engrg., 139:289-314, 1996.

[34] M. Stolarska, D. L. Chopp, N. Moës, and T. Belytschko. Modelling Crack Growth by Level Sets and the Extended Finite Element Method. International Journal for Numerical Methods in Engineering, 51(8):943-960, 2001.

[35] SF.L. Stazi, E. Budyn, J. Chessa, and T. Belytschko. An extented finite element method with higher-order elements for curved cracks. Computational Mechanics, 31:38-48, 2003.

[36] A. Legay, H.W. Wang, and T. Belytschko. Strong and weak arbitrary discontinuities in spectral finite elements. International Journal for Numerical Methods in Engineering, 64:991-1008, 2005.

[37] N. Sukumar, D. L. Chopp, E. Béchet, and N. Moës. Three-dimensional non-planar crack growth by a coupled extended finite element and fast marching method. International Journal for Numerical Methods in Engineering, 76(5):727-748, 2008.

[38] A. Huerta, E. Casoni, E. Sala-Lardies, S. Fernandez-Mendez, and J. Peraire. Modeling discontinuities with high-order elements. In ECCM 2010 - Paris, 2010.

[39] G. Legrain, P. Cartraud, Perreard I., and N. Moës. An x-fem and level set computational approach for image-based modelling: Application to homogenization. International Journal for Numerical Methods in Engineering, 86(7):915-934, 2011.

[40] I. Ionescu, N. Moës, P. Cartraud, and M. Beringhier. Application de x-fem et des level-sets à l'homogénéisation de matériaux aléatoires caractérisés par imagerie 
numérique. In 8e COLLOQUE NATIONAL EN CALCUL DES STRUCTURES, 2007.

[41] M. Chiad, W-D. Lian, S. Guessasma, G. Legrain, G. Della Valle, and P. Cartraud. Modélisation numérique par une approche micromécanique du comportement de mousses solides alimentaires. In 9e Colloque National en Calcul des Structures, 2009 .

[42] Patrice Cartraud, Irina Ionescu, Gregory Legrain, and Nicolas Moës. Image-based computational homogenization of random materials using level sets and $\mathrm{x}$-fem. In 5th. European Congress on Computational Methods in Applied Sciences and Engineering (ECCOMAS 2008) - 8th. World Congress on Computational Mechanics (WCCM8), 2008.

[43] W.-D. Lian, G. Legrain, and P. Cartraud. Comparison of two computational approaches for image-based micromechanical modeling. In IV European Conference on Computational Mechanics, 2010.

[44] T. Coupez. Metric construction by length distribution tensor and edge based error for anisotropic adaptive meshing. Journal of Computational Physics, 230(7):2391 $2405,2011$.

[45] Sonia Fernández-Méndez and Antonio Huerta. Imposing essential boundary conditions in mesh-free methods. Computer Methods in Applied Mechanics and Engineering, 193(12-14):1257 - 1275, 2004.

[46] M. Griebel and M.A. Schweitzer. Geometric Analysis and Nonlinear Partial Differential Equations, chapter A particle-partition of unity method. Part V: Boundary conditions, pages 517 - 540. Springer-Verlag, Berlin, 2002.

[47] G. Legrain, R. Allais, and P. Cartraud. On the use of the extended finite element method with quatree/octree meshes. International Journal for Numerical Methods in Engineering, 86(6):717-743, 2011.

[48] I. Harari and J. Dolbow. Analysis of an efficient finite element method for embedded interface problems. Computational Mechanics, 46:205-211, 2010.

[49] Rolf Stenberg. On some techniques for approximating boundary conditions in the finite element method. Journal of Computational and Applied Mathematics, 63(13):139-148, November 1995.

[50] H. Mourad, J.E. Dolbow, and I. Harari. A bubble-stabilized finite element method for dirichlet constraints on embedded interfaces. International Journal for Numerical Methods in Engineering, 69:772-793, 2007.

[51] A. B. Tran, J. Yvonnet, Q-C. He, C. Toulemonde, and J. Sanahuja. A multiple level set approach to prevent numerical artefacts in complex microstructures with nearby inclusions within xfem. International Journal for Numerical Methods in Engineering, 85(11):1436-1459, 2011. 
[52] Howard E. Hinnant. A fast method of numerical quadrature for p-version finite element matrices. International Journal for Numerical Methods in Engineering, 37(21):3723-3750, 1994.

[53] Barna Szabó and Ivo Babuška. Finite Element Analysis. John Wiley \& Sons, New York, 1991.

[54] J. J. Ródenas-garcía, E. Nadal, J. Albelda, and F. J. Fuenmayor Fernández. Hadaptive refinement based on a nearly statically admissible recovered stress field for meshes independent of the geometry. In IV European Conference on Computational Mechanics, 2010.

[55] Pierre Arnaud Raviart and Jean-Marie Thomas. Introduction á l'analyse numérique des équations aux dérivées partielles. Dunod, Paris, 1998.

[56] J.-F. Remacle, E. Marchandise, N. Chevaugeon, and C. Geuzaine. Efficient visualization of high order finite elements. International Journal for Numerical Methods in Engineering, 69(4):750-771, 2007.

[57] Ibanez L., Schroeder W., Ng L., and Cates J. The ITK Software Guide. Kitware, Inc., 2005 edition, 2005.

[58] T. Kanit, S. Forest, I. Galliet, V. Mounoury, and D. Jeulin. Determination of the size of the representative volume element for random composites: statistical and numerical approach. International Journal of Solids and Structures, 40(1314):3647-3679, 2003.

[59] Toufik Kanit, Franck N'Guyen, Samuel Forest, Dominique Jeulin, Matt Reed, and Scott Singleton. Apparent and effective physical properties of heterogeneous materials: Representativity of samples of two materials from food industry. Computer Methods in Applied Mechanics and Engineering, 195(33-36):3960-3982, 2006.

[60] Martin Ostoja-Starzewski. Material spatial randomness: From statistical to representative volume element. Probabilistic Engineering Mechanics, 21(2):112-132, 2006.

[61] K. Dréau, N. Chevaugeon, and N. Moës. Higher order x-fem for curved cracks. In IV European Conference on Computational Mechanics, 2010. 